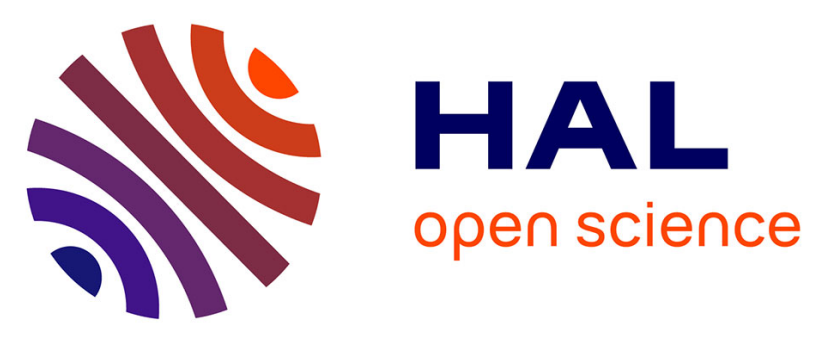

\title{
Aggregation of Antibody Drug Conjugates at Room Temperature: SAXS and Light Scattering Evidence for Colloidal Instability of a Specific Subpopulation
}

B. Frka-Petesic, D. Zanchi, N. Martin, S. Carayon, S. Huille, Christophe Tribet

\section{To cite this version:}

B. Frka-Petesic, D. Zanchi, N. Martin, S. Carayon, S. Huille, et al.. Aggregation of Antibody Drug Conjugates at Room Temperature: SAXS and Light Scattering Evidence for Colloidal Instability of a Specific Subpopulation. Langmuir, 2016, 32 (19), pp.4848-4861. 10.1021/acs.langmuir.6b00653 . hal-01510479

\section{HAL Id: hal-01510479 \\ https://hal.sorbonne-universite.fr/hal-01510479}

Submitted on 19 Apr 2017

HAL is a multi-disciplinary open access archive for the deposit and dissemination of scientific research documents, whether they are published or not. The documents may come from teaching and research institutions in France or abroad, or from public or private research centers.
L'archive ouverte pluridisciplinaire HAL, est destinée au dépôt et à la diffusion de documents scientifiques de niveau recherche, publiés ou non, émanant des établissements d'enseignement et de recherche français ou étrangers, des laboratoires publics ou privés. 


\title{
Aggregation of antibody drug conjugates at room
}

\section{temperature: SAXS and light scattering evidence for}

\section{colloidal instability of a specific subpopulation.}

\author{
B. Frka-Petesic, ${ }^{1+}{ }^{\dagger}$ D. Zanchi,${ }^{1,3}$ N. Martin,${ }^{1+}$ S. Carayon,${ }^{2}$ S. Huille, ${ }^{2}$ C. Tribet. ${ }^{1, *}$
}

1. Ecole Normale Supérieure-PSL Research University, Département de Chimie, Sorbonne Universités - UPMC Univ Paris 06, CNRS UMR 8640 PASTEUR, 24 rue Lhomond, 75005 Paris, France.

2. SANOFI R\&D, Analytics \& Formulation Department, Global Biologics, 13 quai Jules Guesde - BP 14 - 94403 - Vitry-sur-Seine, France.

${ }^{3}$ Université Paris Diderot-Paris 7, 5 rue Thomas Mann, 75013 Paris, France.

\section{ABSTRACT}

Coupling an hydrophobic drug onto monoclonal antibodies via Lysine residues is a common route to prepare antibody-drug conjugates (ADC), a promising class of biotherapeutics. But a few chemical modifications on protein surface often increases aggregation propensity, without clear understanding of the aggregation mechanisms at stake (loss of colloidal stability, self- 
assemblies, denaturation...), and the statistical nature of conjugation introduces polydispersity in the ADC population, which raises questions on whether the whole ADC population becomes unstable. To characterize the average interactions between ADC, we monitored small angle Xray scattering in solutions of monoclonal $\operatorname{IgG1}$ human antibody drug conjugate, with average degree of conjugation of 0,2 , or 3 drug molecules per protein. To characterize stability, we studied kinetics of aggregation at room temperature. Intrinsic Fuchs stability ratio of the ADC was estimated from the variation over time of scattered light intensity and hydrodynamic radius, in buffers of varying $\mathrm{pH}$, and at diverse sucrose $(0 \%$ or $10 \%)$ and $\mathrm{NaCl}(0$ or $100 \mathrm{mM})$ concentrations. We show that stable ADC stock solutions became unstable upon $\mathrm{pH}$ shift, well below the $\mathrm{pH}$ of maximum average attraction between IgGs. Data indicates that aggregation can be ascribed to a fraction of ADC population usually representing less than $30 \mathrm{~mol} \%$ of the sample. In contrast to the case of (monodisperse) monoclonal antibodies, our results suggest that a poor correlation between stability and average interaction parameters should be expected as a corollary of dispersity of ADC conjugation. In practice, the most unstable fraction of the ADC population can be removed by filtrations, which affects remarkably the apparent stability of the samples. Finally, the lack of correlation between the kinetic stability and variations of the average inter-ADC interactions is tentatively attributed to the uneven nature of charge distributions and the presence of patches on the drug-modified antibodies. 


\section{Introduction.}

Aqueous solutions of therapeutic proteins and particularly of antibodies are now competing with small molecular drugs in the treatment of cancers, autoimmune diseases, and several other human diseases, to such an extent that monoclonal antibodies (mAb) became key players in the research for new drugs. ${ }^{1,2}$ The versatility of molecular design of mAb now fuels growing interest for the study of patentable modifications of approved antibodies. In this context, antibodyconjugated particles ${ }^{3}$ or antibody-drug conjugates (ADC), prepared by coupling mAbs with small drug molecules, ${ }^{4}$ appear as one of the most promising emerging class of biotherapeutics, as they can ensure improved targeting of drugs. ADC may be either randomly modified, or prepared by single, site-specific coupling yielding well-defined compounds with typically one or a few drug molecule per protein. However, biomedical acceptance of new therapeutic proteins, even when they are derived from a formerly approved parented antibody, requires to address stability issues and in particular to alleviate aggregation. In the case of non-conjugated monodisperse mAbs, these requirements are generally fulfilled by decreasing possible attractive interactions between pairs of proteins and between proteins and interfaces, which translates to 1) a proper choice of the protein sequence, buffer conditions and excipients, ${ }^{5,6}$ and 2) limiting contacts with hydrophobic interfaces. ${ }^{7,8}$ But the development of ADC has given rise to specific concerns related to conjugation-dependent loss of stability, specifically when ADCs are conjugated to highly hydrophobic molecules. Open questions about ADC stability include 1) the role of conjugated drugs in ADC-ADC interactions, and in particular in inter-ADC attractions and 2) the possible role of the polydispersity introduced by the chemical coupling (e.g. how sensitive are ADC interactions to the number and distribution of a few chemical modifications on the surface of the protein?). Compared to the parent $\mathrm{mAb}$, a more complex interplay emerges accordingly 
between heterogeneity of $\mathrm{ADC}$, conformational stability, oligomerization and aggregation propensity. In particular, aggregation-prone ADC may correspond to a subfraction of the whole population of conjugated antibodies.

We report here a study of ADC aggregation rate as a function of buffer condition, comparing parent $\mathrm{mAb}$ (ADC0) with mAb carrying on average $\sim 2$ (ADC2) or $\sim 3$ (ADC3) drug molecules. We aimed to identify whether formation of aggregates correlated with inter-ADC average interaction. Aqueous solutions of $\mathrm{ADC}$ in buffers of varying $\mathrm{pH}$, in the presence or not of sucrose and/or $\mathrm{NaCl}$, were used to modulate either long-range Coulomb interactions, or shortrange hydration-related interactions between proteins. The colloidal stability of ADC solutions was monitored by variation over time of the mean scattered light intensity, and measurement of hydrodynamic radii by dynamic light scattering (DLS). Kinetics data were fitted to a slow aggregation model yielding apparent Fuchs stability ratios (based on Smoluchowski's theory, and recently used for characterization of protein aggregation, ${ }^{9}$ including for antibody ${ }^{10,}{ }^{11}$ ). Small angle X-ray scattering (SAXS) enabled us to estimate the influence of buffer composition on inter-ADC interactions, and on the shape of ADC molecules. Herein we demonstrate that aggregation involved a $\mathrm{pH}$ and excipient-dependent subpopulation of ADCs. Our results also point out the irrelevance of average interaction parameters (e.g. $2^{\text {nd }}$ virial coefficient) to predict stability conditions of ADC. In contrast, kinetics analysis is better suited to investigate the origin of instability. 


\section{Experimental.}

Aliquoted stock solutions of ADCs.

Water was deionized with a MilliQ Millipore purification system. $\mathrm{NaCl}(99.9 \%$ electrophoretic grade), $\mathrm{HCl}, \mathrm{NaOH}$, Histidine were from Sigma-Aldrich. Monoclonal human IgG1 (characteristic features and sequence detailed in ref. ${ }^{12}$ ), named ADC0 in the following, was provided by Sanofi as 11.6 g. $\mathrm{L}^{-1}$ stock solutions in $10 \% \mathrm{w} / \mathrm{v}$ sucrose, $10 \mathrm{mM}$ Histidine buffer $\mathrm{pH}$ 6.5. Antibody-drug conjugate at two different degrees of conjugation were prepared from ADC0 by random coupling a hydrophobic drug on the amine group of Lysines (cf. patent ${ }^{12}$, reaction in aqueous solution of 3 to 5 molar equivalents of the $N$-hydroxysuccinimide activated ester end of an oligoethylenoxide-containing linker). This procedure yielded mixtures of antibody conjugated molecules having diverse degree of modification around the average one, and a diversity of the distribution of attachment points on the protein. No polishing of this polydispersity was attempted. The reaction mix was filtered (pore size $0.2 \mu \mathrm{m}$ ) to remove antibody aggregates that may form during the reaction. Finally, the solution was ultrafiltered and ADCs were obtained in $10 \mathrm{mM}$ Histidine- $\mathrm{HCl}$ buffer. Sucrose was added to the solution by mixing with $10 \mathrm{mM}$ Histidine-HCl Sucrose $40 \% \mathrm{w} / \mathrm{v}$ buffer in order to achieve a solution of ADC in a $10 \mathrm{mM}$ Histidine-HCl Sucrose $10 \% \mathrm{w} / \mathrm{v}$ buffer, hereafter referred to as HS buffer. Final concentrations were determined by UV absorbance measurements, using extinction coefficients of $1.47 \mathrm{~L} \cdot \mathrm{g}^{-1} \cdot \mathrm{cm}^{-1}$ for $\mathrm{ADC} 0$ (at $280 \mathrm{~nm}$ ), and $0.114 \mathrm{~L} \cdot \mathrm{g}^{-1} \cdot \mathrm{cm}^{-1}$ for the conjugated drug (at $322 \mathrm{~nm}$ ). In solutions of $\mathrm{ADC} 2$ and $\mathrm{ADC} 3$, the protein concentration was determined by subtracting the absorbance at $280 \mathrm{~nm}$ due to the conjugated drug, as follows: [ADC] $\left(\mathrm{g} . \mathrm{L}^{-1}\right)=$ $0.6934 \times\left(\mathrm{OD}_{280}-0.5656 \times \mathrm{OD}_{322}\right)$ where $\mathrm{OD} \lambda$ are absorbances at the wavelength $\lambda$, and 
coefficients were calibrated using standard solutions of known compositions. The degree of conjugation, or Drug-Antibody Ratio (DAR) - i.e. average number of Lysine coupled with the drug per IgG molecule - was estimated from UV-vis absorbance and using a Mw of $150 \mathrm{kDa}$ yielding 2.25 and 3.5 for $\mathrm{ADC} 2$ and $\mathrm{ADC} 3$ respectively, which compares reasonably with the determination of DAR of 2.1 and 3.1 respectively as measured by mass spectroscopy (Sanofi personal communication). One large batch of each stock ADC solution (ADC0, ADC2 and ADC3) was fractionated into $1 \mathrm{~mL}$ aliquots that were kept frozen $\left(-80^{\circ} \mathrm{C}\right)$ until used. Concentrations of stock solutions were 11.6 g.L $\mathrm{L}^{-1}$ (ADC0), 5.9 g.L $\mathrm{L}^{-1}(\mathrm{ADC} 2)$, and $5.8 \mathrm{~g} . \mathrm{L}^{-1}$ (ADC3).

Sample purity was assessed by size exclusion chromatography (HPSEC) using a SEC-Viscotek system equipped with Protein P3000 CLM 3026 columns, UV and RI detectors, at elution rate of $0.5 \mathrm{~mL} \cdot \mathrm{min}^{-1}$ (eluent $\mathrm{Na}_{2} \mathrm{HPO}_{4}-\mathrm{KH}_{2} \mathrm{PO}_{4} 0.45 \mathrm{M} \mathrm{pH}$, and $0.25 \mathrm{M} \mathrm{KCl}$ in acetonitrile:water $20 \%$ $\mathrm{v} / \mathrm{v})$. The main peak of ADC monomers may be flanked by minor peaks of oligomers or aggregates (Figure SI1 in Supporting Info) or degradation peptides. The peptide degradation products were essentially not detected in fresh solutions (Figure SI1 in Supporting Info), and the peak area due to oligomers represented less than $2 \%$ of the area of monomers in ADC0 and $\mathrm{ADC} 2$, or ca. $4 \%$ in $\mathrm{ADC} 3$.

\section{Kinetics (buffer composition, dilution procedures in buffer)}

To prepare solutions, a frozen aliquot of $\mathrm{ADC}(1 \mathrm{~mL})$ was rapidly thawed at room temperature and used within the next two days. Storage buffer $(10 \mathrm{mM}$ Histidine- $\mathrm{HCl} \mathrm{pH} 6.5,10 \% \mathrm{w} / \mathrm{v}$ sucrose) will be noted as "HSB pH 6.5"; the same buffer without sucrose is noted "HB pH 6.5". To minimize osmotic stresses or large $\mathrm{pH}$ heterogeneities upon dilution, the dilute solutions for 
light scattering or SAXS experiments were prepared by mixing one volume (typically $0.5 \mathrm{~mL}$ ) of ADC diluted in HSB pH 6.5 with the same volume of another buffer at pHi (10 mM Histidine$\mathrm{HCl}, 10 \% \mathrm{w} / \mathrm{v}$ sucrose solution, with $\mathrm{pHi}$ adjusted in the window between $\mathrm{pH} 6.5$ and $\mathrm{pH} 10$ prior to mixing). Similarly, ADC solutions at low sucrose content (and/or with $\mathrm{NaCl}$ ) were prepared by first diluting the stock ADC in "HB pH 6.5", down to twice the final concentration,

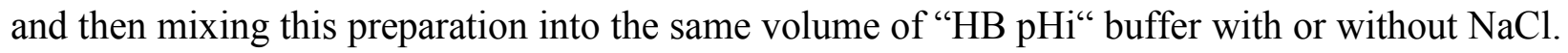
200 ppm sodium azide was also added in the second buffer as a biocide agent. The pH of the final mixtures felt in between 6.5 and $\mathrm{pHi}$, and it was measured with a pHmeter. ADC solutions were filtered through a syringe filter (Anotop 10, pore size $20 \mathrm{~nm}$ or $100 \mathrm{~nm}$, Whatman) either prior to the $1: 1 \mathrm{v} / \mathrm{v}$ mixing (i.e. filtration of the stable dilute solutions of $\mathrm{ADC}$ at $\mathrm{pH} 6.5$ ), or after mixing with the buffer at pHi.

\section{Light scattering}

The aggregation kinetics of ADC at room temperature were assessed by measuring the increase with time of the intensity of the light scattered at a fixed angle of $90^{\circ}$, using a Brookhaven instruments System (BI-200SM goniometer, BI-9000AT correlator, equipped with a $30 \mathrm{~mW}$ laser diode operating at a wavelength of $637 \mathrm{~nm}$ ). Decalin scattering was used as a standard to calibrate the intensity. Initial concentrations of ADC were adjusted (from $\sim 1$ g.L $\mathrm{L}^{-1}$ to 0.005 g.L $\mathrm{L}^{-1}$ ) in order to observe a significant increase of scattered intensity within a few hours. At these dilution conditions, aggregation was sufficiently slow to be negligible during the first 30 s, a time that were necessary to mix solutions and fix the cell in the scattering device. The scattering intensity recorded in solutions at $\mathrm{pH} 6.5$ prior to $\mathrm{pH}$ shift was constant. Subsequently to $\mathrm{pH}$ shift, dilution, and/or addition of sucrose or $\mathrm{NaCl}$, the $\mathrm{ADC}$ solutions were kept at rest at $25^{\circ} \mathrm{C}$. Changes in the scattering intensity were measured as a function of incubation time. Regularly 
during incubation, a 2-minute long record of homodyne intensity-intensity correlation function was performed and size distribution of scatterers were determined by CONTIN and NNLS procedures, to yield apparent Stokes radius, $\mathrm{R}_{\mathrm{h}}$.

\section{Circular Dichroism}

CD spectra of $\mathrm{ADC}$ were recorded at $20^{\circ} \mathrm{C}$ in the $\mathrm{Jasco} \mathrm{J} / 815$ spectrophotometer, using quartz cells of $1 \mathrm{~mm}$ path length. ADC were diluted from their stock solutions into $10 \mathrm{mM} \mathrm{NaH} \mathrm{PO}_{4}$ $\mathrm{NaOH}$ buffer, $10 \%$ sucrose, at $\mathrm{pH}$ adjusted at 6.5, 7.3, or 7.7 (measurements in HS buffer were not possible because of the high UV absorbance of $10 \mathrm{mM}$ Histidine). The specific ellipticity $[\theta]$ was calculated according to the equation:

$$
[\theta]=\frac{0.1 \times \theta e \times M_{R}}{l \times c}
$$

where $\theta e$ is the measured ellipticity in millidegrees, $l$ is the path length $(\mathrm{cm})$, and $c$ is the ADC concentration (in g. $\mathrm{L}^{-1}$ ). $\mathrm{M}_{\mathrm{R}}$ is the mean residue molar mass (we used $\mathrm{M}_{\mathrm{R}}=113.5 \mathrm{~g} \cdot \mathrm{mol}^{-1}$ ).

\section{SAXS}

Synchrotron radiation X-ray scattering was measured on SWING beamline at the SOLEIL synchrotron facility in Saclay, France. The incident beam energy was $12 \mathrm{keV}$. The modulus $q$ of the scattering vector is given by $q=4 \pi \sin (\theta / 2) / \lambda$, where $\theta$ is the scattering angle, $\lambda$ is the X-ray wavelength which was $0.1 \mathrm{~nm}$. In most experiments the sample to detector (Aviex CCD) distance was set to $1817 \mathrm{~mm}$, covering the $q$-range from $0.06 \mathrm{~nm}^{-1}$ to $7 \mathrm{~nm}^{-1}$. Measurements were performed using a thermostated flow-through device for the injection of samples $(30-50 \mu \mathrm{L}$ sample placed in the capillary cell between two air bubbles and flowed continuously during the 
SAXS data acquisition in order to avoid sample degradation by the X-rays). Experiments were temperature-controlled at $25^{\circ} \mathrm{C}$. Typically 40 successive frames of $0.5 \mathrm{~s}$ each were recorded for both samples and ADC-free buffers. Each raw frame was corrected from pixel sensitivity, and angularly averaged. The final spectra and experimental errors were obtained by averaging over all frames and subtracting the buffer spectrum from the sample spectrum. Intensities were scaled to absolute units using the scattering of water.

\section{Computation of distorted mAb structures}

Model distorted mAb structures were generated using the PyMol Molecular Graphics System (Schrödinger, LLC). First, the X-ray crystal structure of Fab regions of the ADC used in this work was superimposed onto the Fab arms of an arbitrarily chosen template mAb monoclonal antibody from RSCB Protein Data Bank (pdb entry 1IGT). The Fc domain of this template murine $\mathrm{mAb}$ was also replaced with a human $\mathrm{Fc}$ (pdb file 3DO3). Structural alignment was performed using the align function within PyMol, by minimizing the RMS deviation of carbon $\alpha$ and backbone atoms of the template and the model. Second, the angle between the Fab and Fc domains were calculated from the dot product between two vectors. Briefly, each vector was defined as the long axis though each Fab or Fc region, each defined as the line passing through the centers of gravity of the immunoglobulin domain folds at the two ends of the Fab and Fc regions (resp. $\mathrm{V}_{\mathrm{L}}-\mathrm{V}_{\mathrm{H}}, \mathrm{C}_{\mathrm{H}} 3-\mathrm{C}_{\mathrm{L}}, \mathrm{C}_{\mathrm{H}} 1-\mathrm{C}_{\mathrm{H}} 2$, see Figure SI2 in SI). The angle between one Fab fragment and the Fc domain was then altered by rotation of the Fab arm in the Fab-Fc plane ( $\pm 10^{\circ}$ angle increments), The origin of rotation was defined at the onset of the hinge region between the Fab and Fc domains (see Figure SI2 in SI). Covalent bonds between Fc and Fab 
domains in the hinge region were artificially broken to preserve the native shape of each domain all along the rotation pathway, and it was ensured that no superimposition occurred between atoms from the $\mathrm{Fab}$ and $\mathrm{Fc}$ domains (which limited the rotation to $\pm 50^{\circ}$ angle).

\section{Electrostatic potential calculations and Lysine substitutions}

Electrostatic calculations were performed on the model $\mathrm{mAb}$ described above (obtained after structural alignment of our model Fab domains and a human Fe domain onto the template 1IGT mAb) with the Adaptive Poisson-Boltzmann Solver (APBS) package (PARSE forcefield, dielectric constant of 80 - resp. 2 - for the protein exterior -resp. interior, $10 \mathrm{mM}$ ionic strength,

probe size of $1.4 \AA) \cdot{ }^{13},{ }^{14}$ The propKa software package ${ }^{15},{ }^{16}$ was used to assign the pKa values of each residue and thus fix their protonation state at $\mathrm{pH}$ 7.8. To model drug conjugation onto $\mathrm{ADC0}$, target Lysine residues were chosen somewhat arbitrarily at the boundary between highlypositive regions and protruding negative poles, or in zwitterionic regions, and the surface potential was recalculated after substitution by neutral Leucine residues assuming preservation of the native structure. The PyMol Molecular Graphics System (Schrödinger, LLC) was used to visualization the isocontour potential $\left( \pm 1 \mathrm{k}_{\mathrm{B}} \mathrm{T} / \mathrm{e}\right)$. The isoelectric points of the substituted structures were also calculated using the propKa software package. 


\section{Results.}

\section{1- Destabilization of ADC upon variation of buffer composition.}

The instability of ADC in various buffers was assessed by recording the scattering intensity of ADC solutions prepared at varying $\mathrm{pH}$ and a fixed protein concentration. Solutions were obtained by diluting a filtered (Anotop 10, $0.1 \mu \mathrm{m}$ pore size) aliquot of stock ADC ( $\sim 6$ g. $\mathrm{L}^{-1}$, in HS buffer $\mathrm{pH} 6.5$ ) with 5-50 fold larger volume of HS buffer $\mathrm{pH} 6.5$, prior to mixing at $\mathrm{t}=0$ (two-fold dilution) with $10 \mathrm{mM}$ Histidine- $\mathrm{HCl}$ buffer of $\mathrm{pH}>6.5$ and $\mathrm{pH}<10$ (final volume $0.8-1 \mathrm{~mL}$, and final $\mathrm{pH}$ measured with a glass electrode, $\mathrm{cf}$. method section). In solutions of $\mathrm{ADC} 2$ and $\mathrm{ADC} 3$, the shift of $\mathrm{pH}$ immediately resulted in an increase of light scattering intensity, indicating that $\mathrm{pH}$ shift triggered aggregation (Figure 1). Species of diameter $>100 \mathrm{~nm}$ were detected by DLS beyond incubation times of a few minutes (Figure 1D), and diameters could reach micrometer range in the case of ADC3. Dilution in buffer of $\mathrm{pH} 6.5$ did not destabilize the ADC, which confirms that it was the change in buffer $\mathrm{pH}$, and not handling conditions, that made ADC unstable. In both ADC2 and ADC3 solutions, the kinetics of aggregation depended markedly on $\mathrm{pH}$, and the maximum rate of the initial intensity variation was reached at $\mathrm{pH} \sim 7.8$. To accurately record intensity variation, it was necessary to optimize the dilution factor in order $i$ ) to avoid that substantial amount of aggregate had formed during the time period required to mix and load the sample in the DLS apparatus $(<20 \mathrm{~s})$, and ii) to measure intensity variations over a time window of several minutes. To this aim, solutions of ADC3 were diluted to a significantly lower concentration $\left(0.03\right.$ g.L $\mathrm{L}^{-1}$ in Figure1B) than solutions of ADC2 (0.10 g. $\left.\mathrm{L}^{-1}\right)$, suggesting a lower stability of the ADC having higher conjugation ratio. Similar measurements conducted with solutions of the non-modified mAb required to use the highest experimental concentration (0.6 g. $\mathrm{L}^{-1} \mathrm{ADC} 0$ in Figure $\left.1 \mathrm{C}\right)$. 
At long times, the scattered intensity reached a plateau that depended on buffer condition (Figure 2A), but aggregates continued to grow in size (continuous increase of apparent hydrodynamic radius as measured by dynamic light scattering, DLS). The plateau intensity was thus reached due to the finite value of the wave-vector transfer, $q=4 \pi / \lambda \sin (\theta / 2)$ : according to RayleighGans-Debye light scattering theory, it is expected that the scattering becomes independent on the size of scattering species when this size reaches the same order of magnitude as the laser wavelength. In this regime, the total intensity varies in proportion to the concentration of scattering species. Here, the diameters of aggregates were above $100 \mathrm{~nm}$, and typically of 300 $\mathrm{nm}-1000 \mathrm{~nm}$ (Figure 1D). Intensities measured at time $>900 \mathrm{~s}$ were thus assumed to be proportional to the concentration of aggregates. Consequently, the observation of a plateau intensity together with continuous increase of apparent radius suggests that the quantity of proteins contributing to the aggregates reaches a maximum, while protein-clusters continuously coalesce and stick to each other. An index of aggregation was defined as the height of the plateau intensity, $I-I_{0}$ (where $I_{0}$ is the initial scattering intensity), normalized by the contribution of IgG monomers to the initial scattering ( $I_{\text {mono }}$, Figure 2$)$. At the $\mathrm{pH}$ of maximum destabilization $(\mathrm{pH} \sim$ 7.8 for $\mathrm{ADC} 2$ and $\mathrm{ADC} 3, \mathrm{pH}<7.5$ for $\mathrm{ADC} 0$ ) and at incubation time long enough to alleviate further significant variation of scattering intensity (here $t>900 \mathrm{~s}$ ), this index reached ca. 3.5 $(\mathrm{ADC} 0), 12(\mathrm{ADC} 2)$, and $>52(\mathrm{ADC} 3)$. The variation of the index of aggregation suggests that protein clusters form faster and/or involve higher amount of protein upon increasing the degree of conjugation of ADC. 

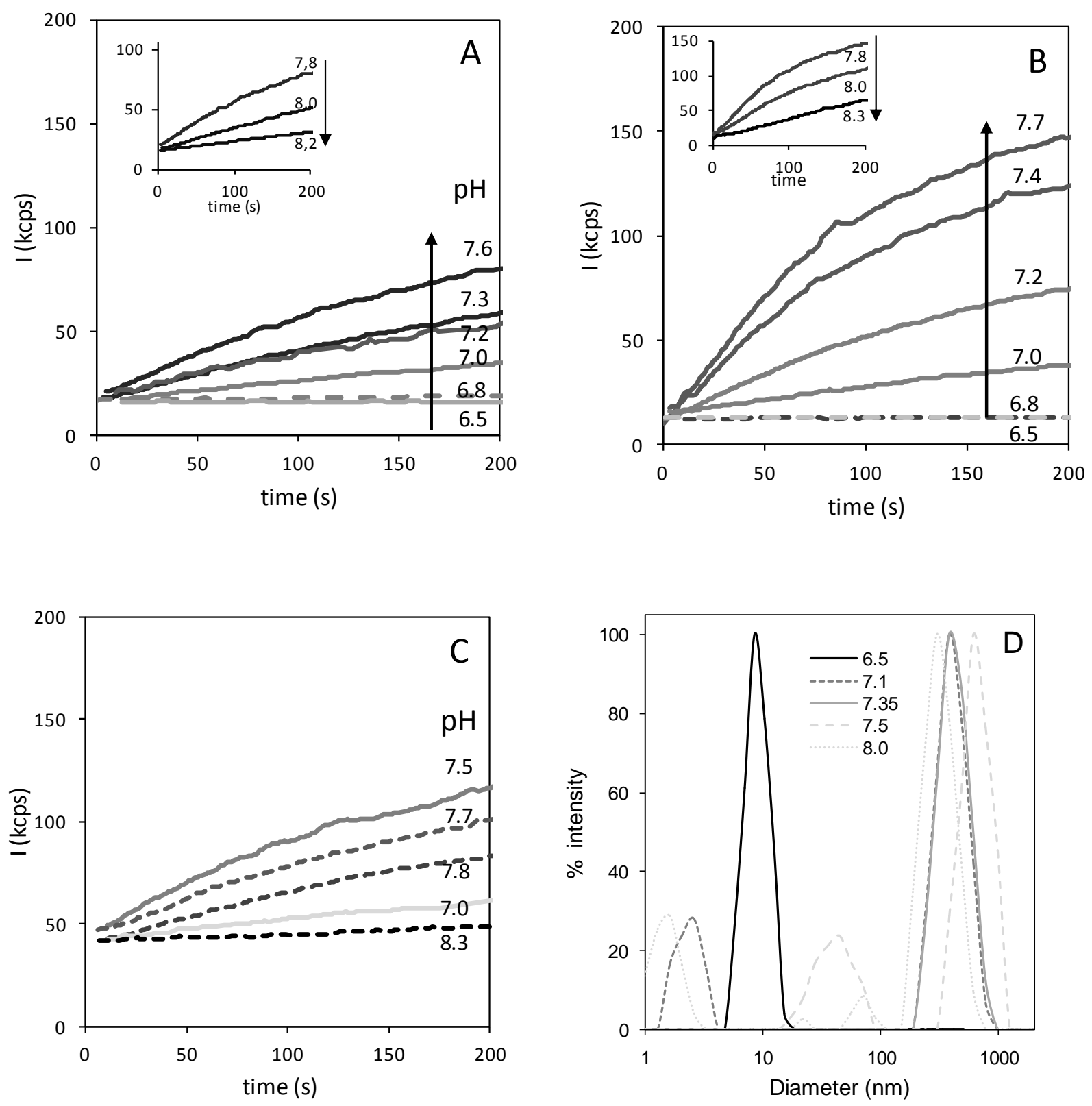

Figure 1. Aggregation kinetics of ADCs triggered by $\mathrm{pH}$ shift, and characterized by light scattering. The $\mathrm{pH}$ shift was obtained by dilution of stock solutions at $\mathrm{pH} 6.5$ in Histidine-HClsucrose buffers , "HS buffer", to a final ADC concentration of (A) 0.1 g.L - $^{-1}$ ADC2 (B) 0.03 g.L - $^{-1}$ ADC3, (C) 0.6 g. $\mathrm{L}^{-1}$ ADC0, (D) examples of size distribution in solutions of ADC2, as determined by NNLS fit of correlations functions (shown in SI Figure SI3) recorded at time 20$30 \mathrm{~min}$ after $\mathrm{pH}$ shift. Reference intensity scattered by decalin $=6 \mathrm{kcps}$, temperature $25^{\circ} \mathrm{C}$, scattering angle $90^{\circ}$. 

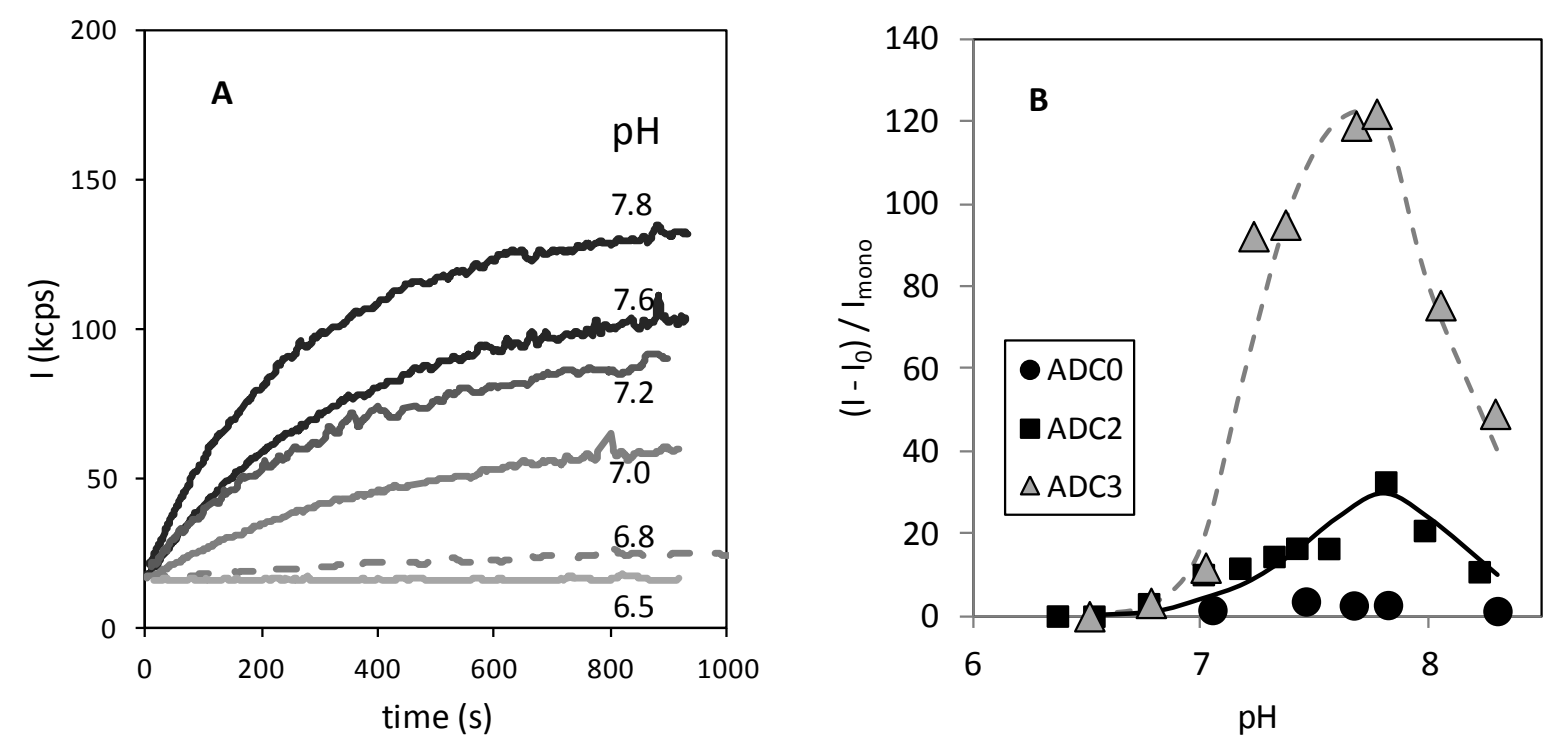

Figure 2. Plateau of scattering intensity reached at long incubation times as a function of $\mathrm{pH}$ in the HS stress buffer. (A) Variation of intensity scattered by a solution of ADC2 (same condition as in Figure 1). (B) $\mathrm{pH}$-dependence of the normalized intensity reached at $\mathrm{t}=500 \mathrm{~s}$, where $\mathrm{I}_{0}$ is the intensity measured at time 0 , and $\mathrm{I}_{\text {mono }}$ is the calculated intensity scattered by ADC monomers in the absence of aggregation. Lines are guide to the eye.

\section{2- Effect of sample filtration.}

Prior to light scattering measurements, filtration of protein solutions was indispensable to remove contributions from dusts and pre-formed aggregates that may be present in stock solutions, and to ensure that only monomers contributed to scattering at time zero. For instance, in the case of $\mathrm{ADC} 0$ (unmodified $\mathrm{mAb}$ ), the aggregation involved a very minor fraction of the whole protein population (cf Figure 2B) that can be formed during freezing/thawing manipulations. Possible consequences of filtration, and specifically adsorption on membranes, 
shall however not be overlooked because of the hydrophobicity of ADC. UV-vis spectra of ADC solutions before and after filtration were clearly indicative of a marked loss of material on the filters (Figure SI5 in SI). Namely, in the first drop $(50 \mu \mathrm{L})$ leaving the filter, the protein concentration in filtrate was decreased to less than a tenth of the initial concentration. Concentration increased gradually with increasing volume passed through the filter (Figure 3A). We estimated by integration over the filtered volumes that a total amount of $60 \mu \mathrm{g}$ of protein was adsorbed before reaching saturation of the filter (Figure 3C). Impact of filtration on the degree of conjugation (DAR) was estimated from the ratio of absorbance at $280 \mathrm{~nm}$ (protein+drug contributions) to absorbance at $322 \mathrm{~nm}$ (drug contribution). It is presented in Figure 3B for a set of filters, showing that Anotop ones did not lead to a significant variation of DAR (5\% is of the order of experimental uncertainty), whereas ADC of higher DAR were preferentially retained on Millex filters. To avoid bias, we fixed as a standard condition of preparation throughout this study: filtration through Anotop membranes that were presaturated by passing $100 \mu \mathrm{L}$ of the protein solution, which ensured that in the absence of aggregates, $[\mathrm{ADC}]$ in filtrates was equal within uncertainty to $[\mathrm{ADC}]$ prior to filtration. 
A

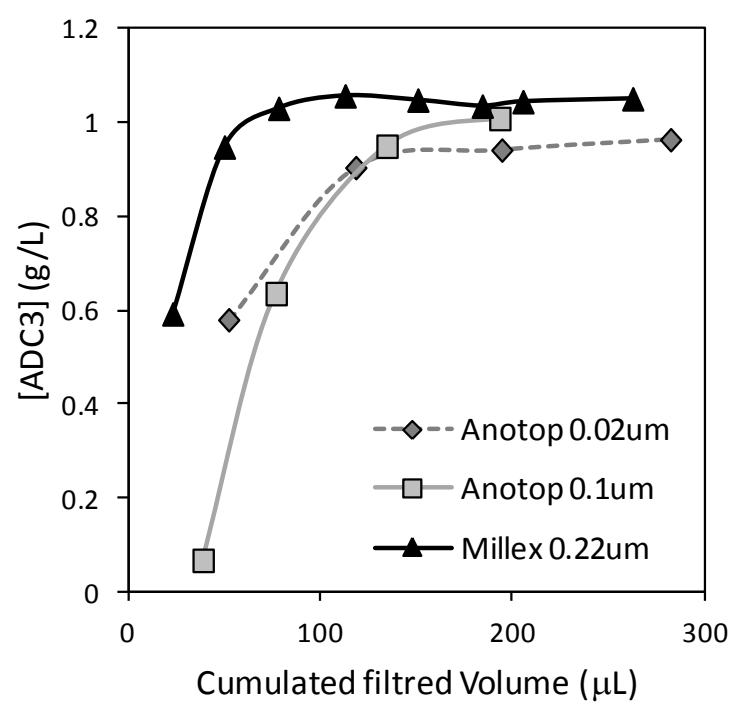

C

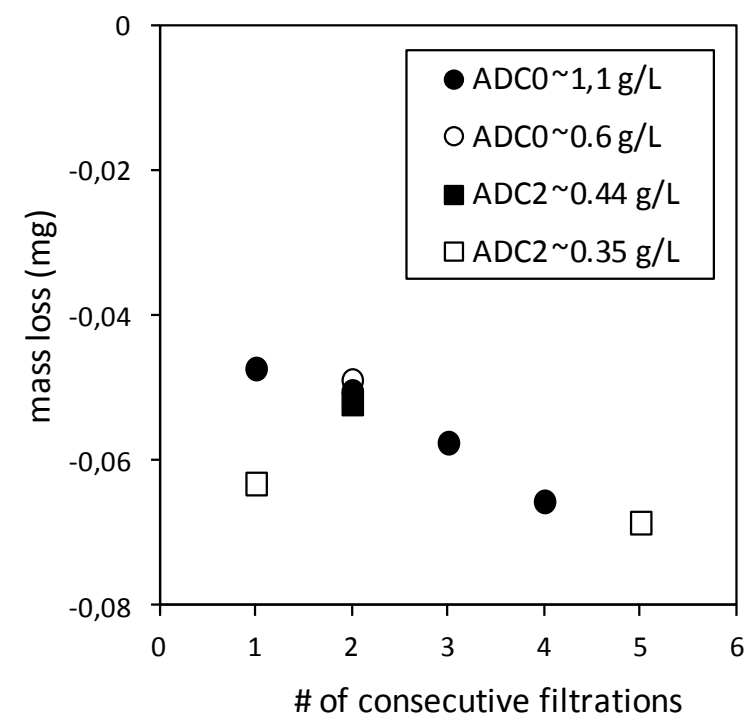

B

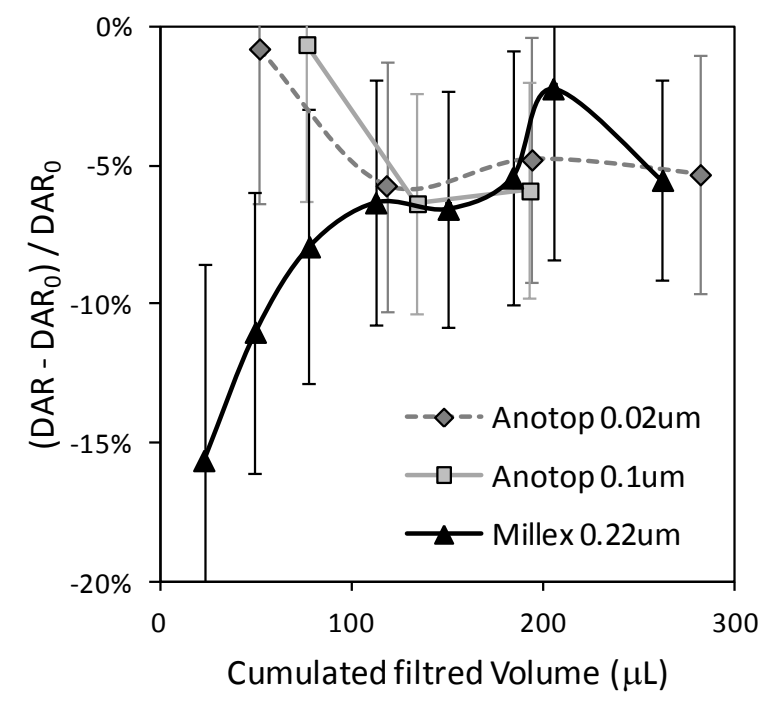

Figure 3. Variation of composition of filtrates of ADC solutions at $\mathrm{pH} 6.5$ due to adsorption on filters. (A) concentration of ADC3 in filtrate vs filtered volume of a stock solution at 1 g. $\mathrm{L}^{-1}$, (B) DAR in filtrate as determined by spectrophotometry (same solution as in (A)), (C) Total mass adsorbed on Anotop $0.02 \mu \mathrm{m}$ filter during repetitive filtration steps through the same filter (sample volume of $1 \mathrm{~mL}$ was fully filtered at each step, ADC concentrations in samples quoted in the Figure). 
Filtration through presaturated filters was used to estimate the effect of aggregation on the DAR of soluble fractions. To this aim, aliquots of the stock solutions of ADC (unfiltered) were diluted into Histidine-sucrose buffers at various final pHs, and incubated for $18 \mathrm{~h}$. UV-vis spectra of solutions were measured immediately after dilution, then at time $18 \mathrm{~h}$ prior and after filtration (Anotop 10, $100 \mathrm{~nm}$ pore size). Several successive spectra were collected during the dropwise filtration (every ca. $150 \mu \mathrm{L}$ ) until no variation could be detected between two successive measurements. The composition of filtrates was thus considered to reflect the fraction of soluble (monomer + oligomers) ADC. Representative $\mathrm{pH}$ conditions are reported in Table 1 . In the majority of samples, turbidity was low prior to filtration enabling accurate measurements of [ADC] and DAR (Figure 4A), except with ADC3 at pH >7.8 (Figure 4B). Absorbance of filtrates at $280 \mathrm{~nm}$ was systematically lower than before filtration, suggesting that a fraction of ADC formed aggregates with diameters $>100 \mathrm{~nm}$. Absorbance at $280 \mathrm{~nm}$ and $322 \mathrm{~nm}$ were used to determine apparent DAR (see method section). The decrease of DAR in filtrates as compared to its initial value before filtration, suggested that ADC with higher DAR aggregated preferentially. Small decrease of DAR in the (typically predominant) filterable fraction corresponded to a marked increase of DAR in aggregates, well above 2 and typically $>3.5$ (i.e. beyond the apparent DAR measured in ADC3 stock solution). To estimate the average DAR reached in the aggregated fraction (i.e. retained on the filter), we calculated in Table 1 the ratio ([drug $]_{\text {unfiltered }}$ $\left.[\mathrm{drug}]_{\text {filtered }}\right) /\left([\mathrm{ADC}]_{\text {unfiltered }}-[\mathrm{ADC}]_{\mathrm{filtered}}\right)$ where $[\mathrm{drug}]$ and $[\mathrm{ADC}]$ are molar concentrations. These measurements confirmed the poorer stability of antibody-drug conjugates having the highest DAR values in the polydisperse population of ADC. 

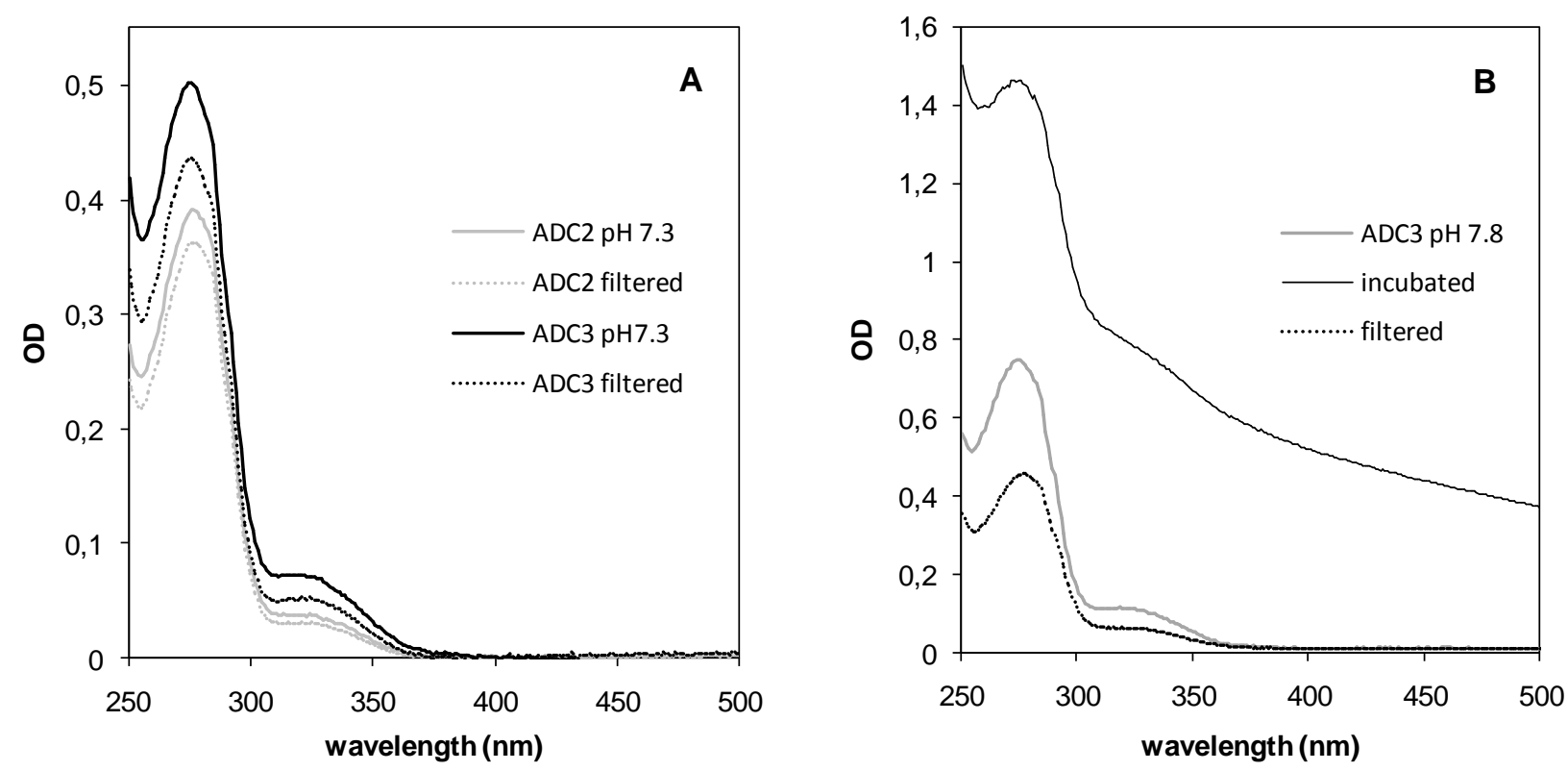

Figure 4. UV-vis. absorption spectra of solutions of ADC incubated for $18 \mathrm{~h}$ in HS buffer prior or after filtration on Anotop 10 (100 nm pore size).(A) pH 7.3 and $0.25 \mathrm{~g} . \mathrm{L}^{-1} \mathrm{ADC}$, or $0.3 \mathrm{~g} . \mathrm{L}^{-1}$ ADC3 ; (B) pH 7.8 and 0.5 g.L $\mathrm{L}^{-1}$ ADC3; "incubated" stands for the unfiltered sample at time $18 \mathrm{~h}$ after mixing in the stress buffer.

Table 1. DAR variation and \% filter-removed aggregates in solutions of ADC as determined by UV-vis spectrophotometry at time $18 \mathrm{~h}$ after $\mathrm{pH}$ shift (incubation at room temperature, unfiltered solution or filtrates from protein-saturated Anotop 10, see text).

\begin{tabular}{|c|c|c|c|c|c|c|c|c|}
\hline \multirow[b]{2}{*}{$\mathbf{p H}$} & \multicolumn{4}{|c|}{ ADC2 (0.25 g.L-1) } & \multicolumn{4}{|c|}{$\operatorname{ADC3}\left(0.5\right.$ or 0.3 g.L $\left.\mathrm{L}^{-1}\right)$} \\
\hline & 6.8 & 7.2 & 7.8 & 8.2 & 6.8 & 7.3 & 7.8 & 8.2 \\
\hline \%filtered out ${ }^{\text {a }}$ & $6 \%$ & $3.0 \%$ & $8 \%$ & $2.5 \%$ & $7 \%$ & $13 \%$ & $40 \%$ & $32 \%$ \\
\hline DAR unfiltered & 2.35 & 2.25 & 2.30 & 2.15 & 3.40 & 3.55 & $\mathrm{nd}^{\mathrm{c}}$ & $\mathrm{nd}^{\mathrm{c}}$ \\
\hline DAR in filtrate & 2.15 & 2.33 & 2.17 & 2.0 & 3.20 & 3.20 & 2.90 & 2.85 \\
\hline DAR aggregates ${ }^{b}$ & $\sim 5.3 \pm 0.7$ & nd & $3.8 \pm 0.4$ & nd & $4.4 \pm 0.5$ & $5.5 \pm 0.2$ & $4.6 \pm 0.1$ & $5.2 \pm 0.1$ \\
\hline
\end{tabular}
concentration prior to filtration and $[\mathrm{ADC}]_{\mathrm{filt}}$ in the filtrate.

b. see text for the definition. Uncertainty was estimated using an experimental error of \pm 0.5 $\mathrm{mDO}$ for the absorbances, which introduces uncertainties of $\sim 0.05-0.07$ in the DAR. "nd" indicates that DAR in aggregates could not be reliably estimated due to low \% aggregates.

${ }^{c}$ not measurable, due to high turbidity (see Figure 4B) 
Finally, to assess the role of nascent clusters in ADC instability, we considered the effect of a filtration performed just after the $\mathrm{pH}$ shift. In this alternative protocol, aggregates that were rapidly formed upon dilution in the running buffer were removed at time $<60$ s, i.e. after the application of the $\mathrm{pH}$ stress conditions. Data clearly point to an important impact of filtration on aggregation kinetics. In contrast to the slow increase of scattered intensity by mixtures that were filtered prior to $\mathrm{pH}$ shift (see section above), filtration within $60 \mathrm{~s}$ after mixing the stock ADC into the Histidine-HCl stress buffer led to fully stable solutions. Here, scattered intensity and measured average diameters were constant for days, irrespective of $\mathrm{pH}$ (ADC0 and ADC2 in Figure SI4 in SI). Hydrodynamic diameters were compatible with the preservation of monomers of the protein in these samples. This lack of aggregation in post-stress filtered solutions indicates that removal of aggregates formed at short time cancels further aggregation. The residual fraction of soluble ADC in filtrate is thus stable. Depending on $\mathrm{pH}$, it may represent a $70 \%-90 \%$ of the whole initial population (see below \% aggregates in Table 2)

\section{3- Kinetics stability in different buffer conditions.}

In order to access to a quantitative index of stability as a function of buffer conditions, the kinetics data were fitted to the model of slow colloid aggregation developed by Smoluchowski and recently used to analyze "cold-set" protein aggregation ${ }^{9,10,11}$ ). At the onset of irreversible aggregation of colloids, this theory predicts that the average molar mass varies in proportion to time and initial particle concentration. This variation is reflected in the linear increase of scattered intensity, given in Equation 2, as long as the form factor of aggregates does not modulate the signal. Equation 2 shall thus be valid at short time, until aggregates are larger than 
$50-100 \mathrm{~nm}$ (and as long as deviation from linearity is negligible, which interestingly, goes much beyond the formation of protein dimers).

$$
I(t)=I(0)\left(1+\frac{k[A D C]}{2 W} t\right) \quad \text { Eq. } 2
$$

where $W$ is the Fuchs stability ratio, $t$ is the incubation time, $[A D C]$ the concentration of protein participating to aggregation, $k$ a constant taken here to be equal to $8 k_{B} T / 3 \eta$, with $\eta$ the buffer viscosity, $k_{B}$ the Boltzmann constant and $T$ the temperature. The validity of equation 2 was experimentally assessed by measurements at increasing ADC concentrations in similar conditions as described in section 1, and Figures 1 and 2. Figure 5 (and Figure SI6 in SI) zooms in the initial increase of scattered light by solutions of ADC initially kept at $\mathrm{pH} 6.5$ (Anotopfiltered) and rapidly mixed at time zero with an Histidine-sucrose buffer at a higher $\mathrm{pH}(1: 1$ volume ratio). In the range of concentrations shown, the initial slope of variation of intensity vs time increased from almost zero (a slope $<0.01 \mathrm{kcps} / \mathrm{s}$ was of the order of noise produced by dusts or bubbles after mixing) to $>60 \mathrm{kcps}$ in $100 \mathrm{~s}$. The rate of intensity variation appeared to increase in proportion to the square of [ADC] (insets in Figure 5 and SI6 in SI) as expected from equation 2. Two practical points are however important to consider. First, scattering suffers from a high sensitivity to small amount of large particles, dust or microbubbles, that may affect the quality of data. They appeared as abrupt spikes of intensity with short durations (1s-3s). Obvious spikes were removed from raw data prior to fitting. Second, the scattering at short times can be matched to equation 2 only if theoretical expectation of the absence of aggregates at time zero is fulfilled, which implies that the initial scattered intensity must be equal to scattering from buffer $+\mathrm{ADC}$ monomers. For instance, measurements at high $[\mathrm{ADC}]$ did enable linear extrapolations, but with lack of consistency in term of the value of initial intensity. At "too" high [ADC], a 
significant amount of aggregation occurred within the mixing time and/or sampling time (1s sampling and a few seconds needed to mix and then relax the agitation). When practiced in the relevant concentration conditions (as in Figure 1 and 5, ADC2 $<0.3 \mathrm{~g} / \mathrm{L}$ and ADC3 $<0.03 \mathrm{~g} / \mathrm{L}$ ) contributions of monomers of ADC to scattering were low $(<3 \mathrm{kcps})$ and extrapolated initial intensities were close to the intensity scattered by the solution of monomers. In Figure 5, extrapolation of measured intensities to time zero did generally not exceed $20 \%$ increase compared to scattering from the buffer+ADC, which indicates that no, or only limited aggregation, took place before we started recording.

A

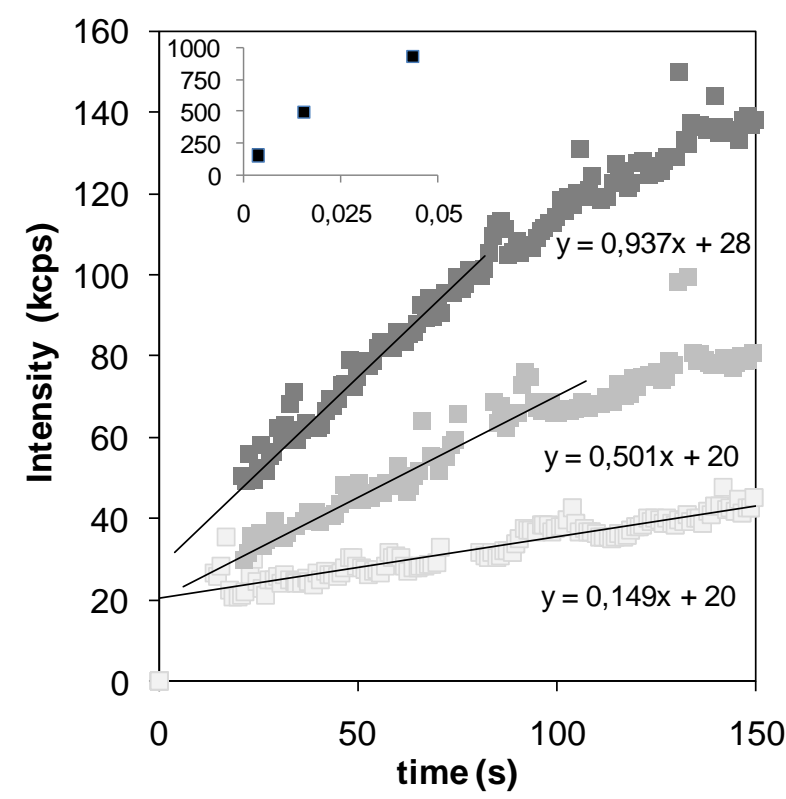

B

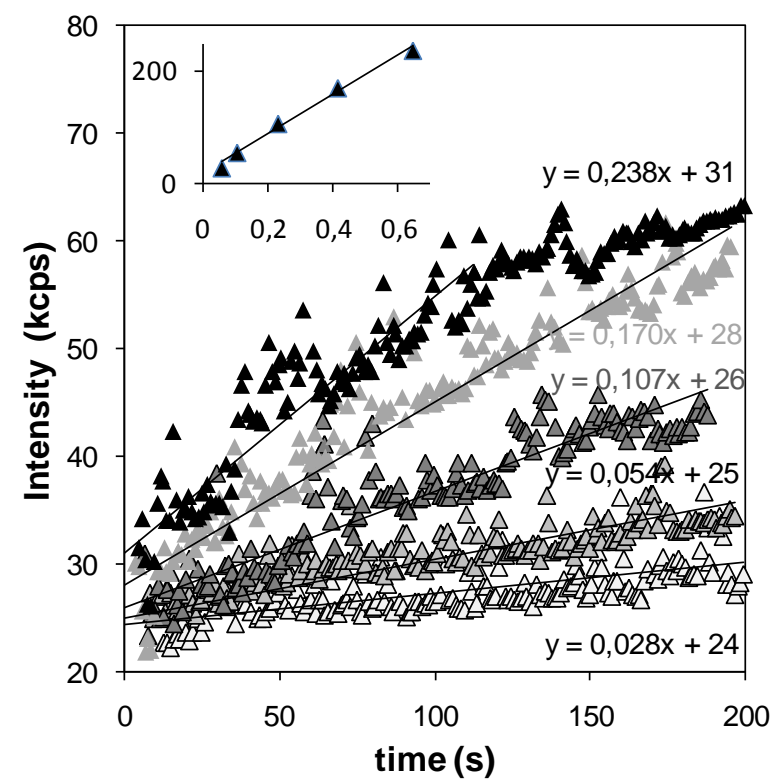

Figure 5. Variation with $\mathrm{ADC}$ concentration of the aggregation kinetics triggered by $\mathrm{pH}$ shift at $\mathrm{t}=0$, and characterized by light scattering. Experimental conditions were the same as in Figure 1.(A) $\mathrm{ADC} 2$ at $\mathrm{pH} 8.0 \pm 0.05$ and $0.06,0.125,0.21$ g. $\mathrm{L}^{-1}$; (B) $\mathrm{ADC} 3$ at $\mathrm{pH} 8.0 \pm 0.05$ and $0.08,0.01,0.015,0.02,0.025 \mathrm{~g} . \mathrm{L}^{-1}$. Lines are fits to linear variations of the intensity vs time, with corresponding equation shown in the Figure. Insets plot the fitted slopes $(\times 1000)$ vs the square of $[\mathrm{ADC}](\times 1 \mathrm{ADC} 2$ or $\times 1000 \mathrm{ADC} 3)$. 


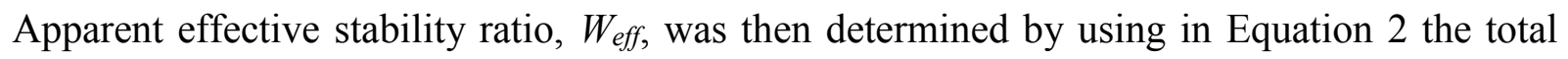
concentration of $\mathrm{ADC}$ (aggregation-prone fraction assumed to be 100\%). Although this calculation overestimated the amount of ADC truly entering in the aggregation process, it gave an average index of the kinetics stability. Not surprisingly, $W_{\text {eff }}$ of the different ADC in the same buffer followed the order $\mathrm{ADC} 3<\mathrm{ADC} 2<\mathrm{ADC} 0$ (Figure 6). $W_{\text {eff }}$ was highly sensitive to $\mathrm{pH}$ (variation of $W_{\text {eff }}$ on more than 5 decades), suggesting an important role of the protein ionization state. $W_{\text {eff }}$ changed to a lesser extent (variation by a factor of 100) with adding $100 \mathrm{mM} \mathrm{NaCl}$ or sucrose in buffers at fixed $\mathrm{pH}$ (Figure 6B). The increasing stability ratio upon increasing the ionic strength near $\mathrm{pH}$ of minimum stability $(7<\mathrm{pH}<8)$, indicates the presence of coulomb attractions between unstable ADC. The decrease of the stability ratio in the presence of sucrose (an osmolyte favoring protein compactness and folding) suggests that conformational stability was not the main origin of aggregation. The conventional trend associated with preferential hydration of proteins is that the presence of sucrose facilitates interprotein contacts, which is here reflected by faster aggregation. ${ }^{17}$ 


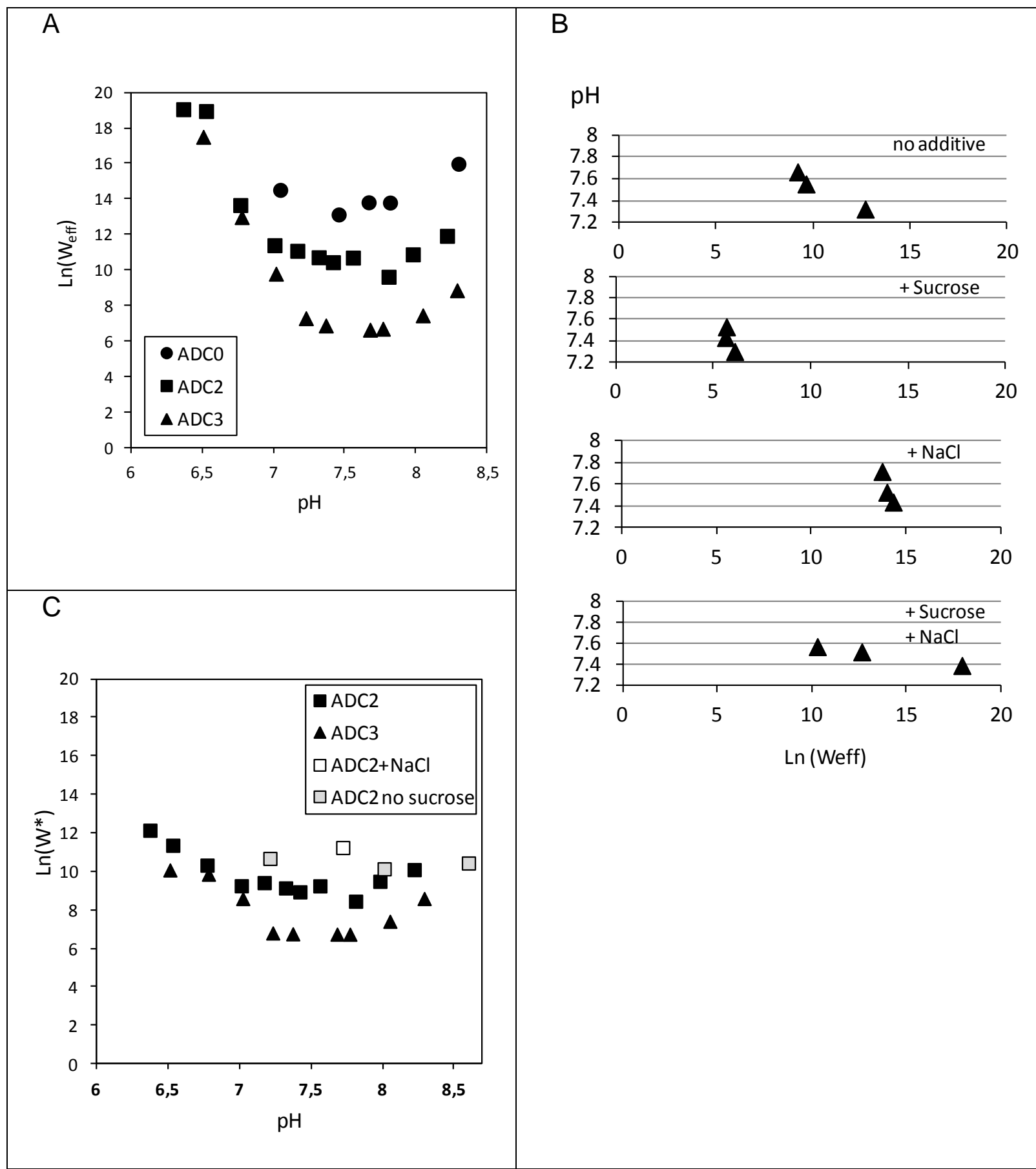

Figure 6. Fuchs stability ratio of ADCs determined from kinetic data in Figure 1 and Equation 2. (A) $W_{\text {eff }}$ in buffer with no $\mathrm{NaCl}$ and with sucrose, calculated using the total ADC concentration in eq. 2, (B) $W_{\text {eff }}$ of ADC3 determined in buffer containing or not sucrose $(0 ; 0.3 \mathrm{M})$, and $\mathrm{NaCl}$ $(0 ; 100 \mathrm{mM})$ in addition to $10 \mathrm{mM}$ Histidine- $\mathrm{HCl}(\mathrm{C}) W^{*}=W_{\text {eff }} \times$ (fraction of ADC aggregated at long time, as estimated from data in Figure 2). 
Plateaus of intensity varied in reverse order compared to the kinetic stability ratio $W_{\text {eff }}$ (ADC3 $>\mathrm{ADC} 2>\mathrm{ADC} 0$ ), suggesting that lower (average) stability as estimated from $W_{\text {eff }}$ was associated with larger fraction of unstable ADC. To define a stability ratio that is not averaged over the whole population, and is representative of the fraction of unstable ADCs, we calculated $W^{*}$ using in Equation 2 the concentration of the unstable fraction, which reads $W^{*}=W_{\text {eff }} \times$ (\%aggregation-prone ADC). The weight-fraction of aggregates was determined in a few reference conditions, by ultracentrifugation of representative solutions and measurement by spectrophotometry of the residual soluble fraction of ADC in supernatants (in concentrated samples to reach measurable absorbance values). Aggregates typically represented a minor fraction of $15 \%-30 \%$ of the total protein concentration (Table 2). Data in Table 2 were used to calibrate the plateau intensities for ADC2 and ADC 3 that were assumed to vary in proportion to the \% aggregated ADC (see Section 1), and thus to calculate $W^{*}$. Interestingly, variation of $W^{*}$ with $\mathrm{pH}, \mathrm{NaCl}$, sucrose, and even difference between $\mathrm{ADC} 2$ and $\mathrm{ADC} 3$ were diminished compared to $W_{\text {eff }}$ (Figure 6C) but the overall impacts of $\mathrm{pH}$ or DAR variation remained qualitatively the same.

Table 2. Weight fraction of insoluble ADC2 measured after 24h incubation in stress buffer at diverse $\mathrm{pH}$.

\begin{tabular}{ccccccc}
\hline buffer & HB+sucrose & HB+sucrose & +sucrose+NaCl & +sucrose+NaCl & HB & HB \\
\hline pH & 7.55 & 8.3 & 7.6 & 8.25 & 7.6 & \multirow{2}{*}{8.05} \\
\hline \% aggregates & 25 & 20 & 13 & 15 & 15 & 35 \\
\hline
\end{tabular}

Initial concentration 0.55 g. $\mathrm{L}^{-1}$; concentration of soluble ADC was determined in supernatants after ultracentrifugation at $200000 \times \mathrm{g}$ for $15 \mathrm{~min}$.; buffer composition was $10 \mathrm{mM}$ Histidine- $\mathrm{HCl}$ ("HB"), with $100 \mathrm{mM} \mathrm{NaCl}(+\mathrm{NaCl})$, and/or $0.3 \mathrm{M}$ sucrose (+sucrose). 


\section{4- SAXS study of average protein-protein interaction and preservation of protein shape.}

Characterization by small angle scattering of IgG1 monomers has been the purpose of several reports, showing nicely that inter-mAb interaction, and intra-mAb shape can be analyzed by SAXS or SANS over a large concentration range (for discussion on form factor and interaction potentials of mAbs see ref. ${ }^{18,19,20,21,22}$ ). In particular, the applicability of SAXS/SANS in monitoring acid-induced, or heat-induced, antibody aggregation was demonstrated. ${ }^{18,22}$ To analyse data, the following assumption are required: weak inter-aggregates interaction $(S(q)$ of aggregates neglected), and additive contributions from aggregates and non-aggregated species, in order to substract the contribution of monomer/oligomers from profiles of aggregate-containing samples (using scattering profiles of filtered, aggregate-free, samples). ${ }^{18}$ Solutions of ADC0 and ADC2 prepared at $\mathrm{pH} 6.5$ predominantly contained monomers of ADC (vide supra) so that SAXS profiles in these conditions were expected to compare with published profiles of mAbs, and to be used as reference for further analysis of aggregate-containing solutions. ADC scattering was measured by SAXS as a function of $\mathrm{pH}$, in buffers supplemented or not with sucrose $\left(0.3 \mathrm{~mol} . \mathrm{L}^{-1}\right)$ and $\mathrm{NaCl}(100 \mathrm{mM})$, i.e. in conditions that yielded aggregation. SAXS spectra recorded at increasing ADC concentrations are shown in Figure 7. To reliably identify the signature of ADC-ADC interactions, we first determined the reference scattering curve reached in the absence of interaction, i.e. the form factor $P(q)$ of $\mathrm{ADC}$ (represented as the full lines in Figures 7). $P(q)$ was calculated by linear extrapolation to the zero-concentration limit of the concentration-normalized scattering intensities. To this aim, the curves in Figure 7 were obtained by subtracting the buffer scattering intensity to raw intensity data, and dividing the result by ADC concentration in $\mathrm{g} / \mathrm{L}$. This calculation introduced a significant noise at $q>0.2 \AA^{-1}$. Alternative normalization uses the assumption of a good matching with Porod law at high q, and 
superimposes the data to the same $q^{-4}$ decrease at $q$ above a threshold (typically $>0.15 \AA^{-1}$, see $\left.{ }^{18,21}\right)$.

Form factors of $\mathrm{ADC} 0$ can be superimposed in the $q$ region $>0.03 \AA^{-1}$ with form factor calculated similarly for $\mathrm{ADC} 2$ at $\mathrm{pH} 6.5$. The absence of variation of $P(q)$, specifically in the high $q$ window, indicates that attachment of the drug on mAb did not significantly affect the overall shape of the protein (which is sensitive to changes of the hinge angle between $\mathrm{Fc}$ and Fab regions, see below).
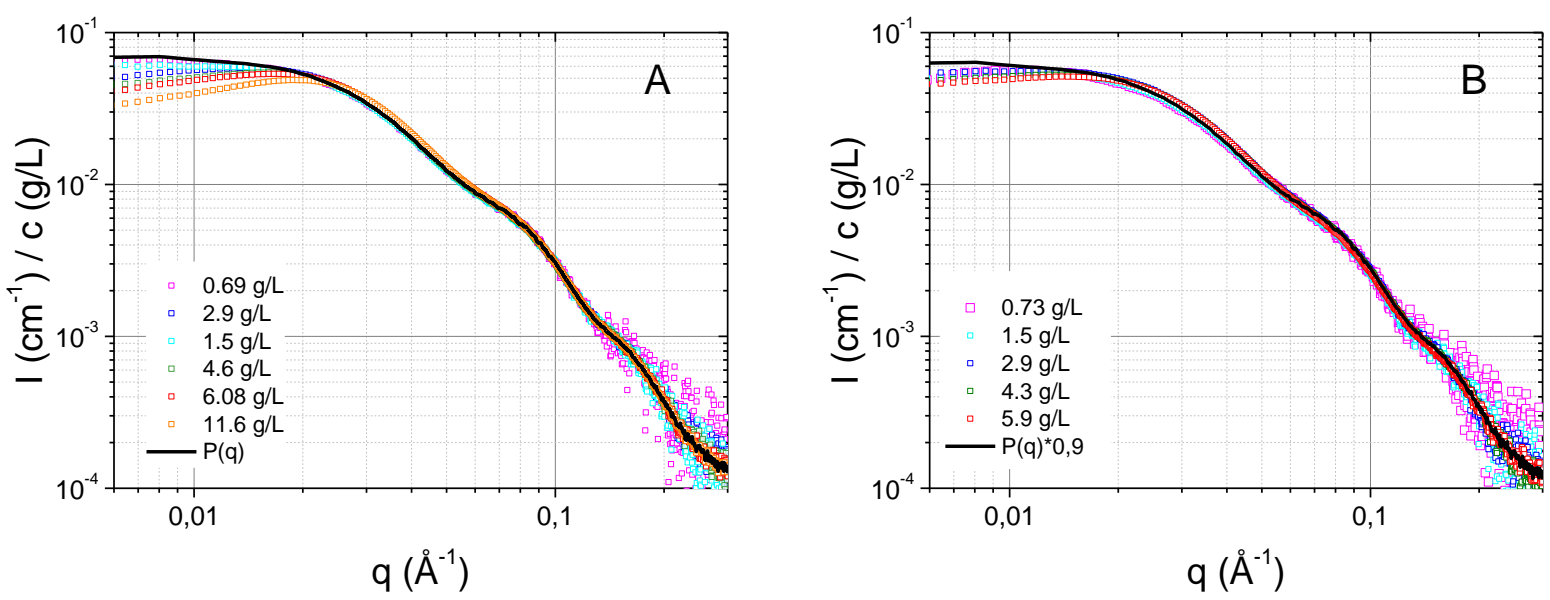

Figure 7. SAXS scattering spectra of $\mathrm{ADC} 0(\mathrm{~A})$ and $\mathrm{ADC} 2(\mathrm{~B})$ at varying $\mathrm{ADC}$ concentration in $10 \mathrm{mM}$ Histidine buffer $\mathrm{pH}$ 6.5. In both figures, $P(q)$ is the form factor of ADC0 calculated by extrapolation of data in Figure 7A to zero concentration.

Using $P(q)$ as a reference curve corresponding to zero average interaction between ADC molecules, the plot of concentration-normalized spectra at a fixed ADC concentration (at 4.35 g. $\mathrm{L}^{-1}$ ) enabled to rank the buffer conditions as a function of the degree of repulsion (low- $q$ drop) or attraction (low- $q$ increase compared to $P(q)$ ). To estimate graphically the transition $\mathrm{pH}$ between attractive and repulsive regimes, we represented $S_{a p p}(q)$, the ratio of the measured 
scattered intensity to $P(q)$ (shifted by a constant factor adjusted between 0.95 and 1.05 in order to account for pipetting errors, and to reach a value of 1 at $q>0.05 \AA^{-1}$, Figure 8). This apparent structure factor deviates from unity at low $q$ due to contributions of correlation in the radial density of ADCs, with upturns corresponding to overconcentration at short interADC distances, i.e. average attractions between the proteins. In the case of ADC0 and ADC2, flat fluctuating values around 1.0 were obtained for $q>0.025 \AA^{-1}$, suggesting that this normalization fairly reports the structure factor. Both samples displayed repulsive patterns at $\mathrm{pH} 6.5$, though with different magnitude of the low- $q$ variation of $S_{a p p}$. Not surprisingly, increasing $\mathrm{pH}$ decreased repulsion and enhanced attraction, presumably because of the gradual charge neutralization of the proteins. In the case of $\mathrm{ADC} 0$, i.e. when aggregates were absent or in negligible fraction, it was possible to measure by light scattering the corresponding virial coefficient that was found to vary from 5.3.10 $0^{-4}$ mol.mL.g $\mathrm{g}^{-2}$ (pH 6.5) down to $-2.1 .10^{-4}$ mol.mL.g ${ }^{-2}$ (pH9.0). Results shown in SI Figure SI7 confirmed the qualitative picture obtained from SAXS: i) the predominance of repulsive interactions between $\mathrm{ADC} 0$ at $\mathrm{pH}<7.8$, ii) a balance of attraction-repulsion at $\mathrm{pH}$ between 7.8 and 8.4 (virial coefficient lower than experimental errors), and inter-ADC0 attractions at $\mathrm{pH}$ 9.0. With increasing DAR, a clear trend was the shift of the balanced (zero) mean interaction to lower pHs. The slight drift of $S_{a p p}$ over the whole $q$ range shown in Figure 8C (ADC3) may indicate in addition a contribution of oligomers that was not further analyzed. Figure $8 \mathrm{D}$ summarizes the results by ranking sample conditions $(\mathrm{pH}, \mathrm{NaCl})$ along a scale indicative of the global magnitude of the low- $q$ variation of $S_{a p p}$. In buffer without $\mathrm{NaCl}$, conditions cancelling interactions are listed as $\mathrm{pH} \approx 8.5(\mathrm{ADC} 0), \mathrm{pH} \leq 8.0(\mathrm{ADC} 2)$, and $\mathrm{pH}>7.0$ (ADC3). Addition of $100 \mathrm{mM} \mathrm{NaCl}$ turns repulsive interaction into attractive ones (pH 6.5) or slightly decreases the magnitude of repulsion (at high pH, Figure SI8 in SI). 

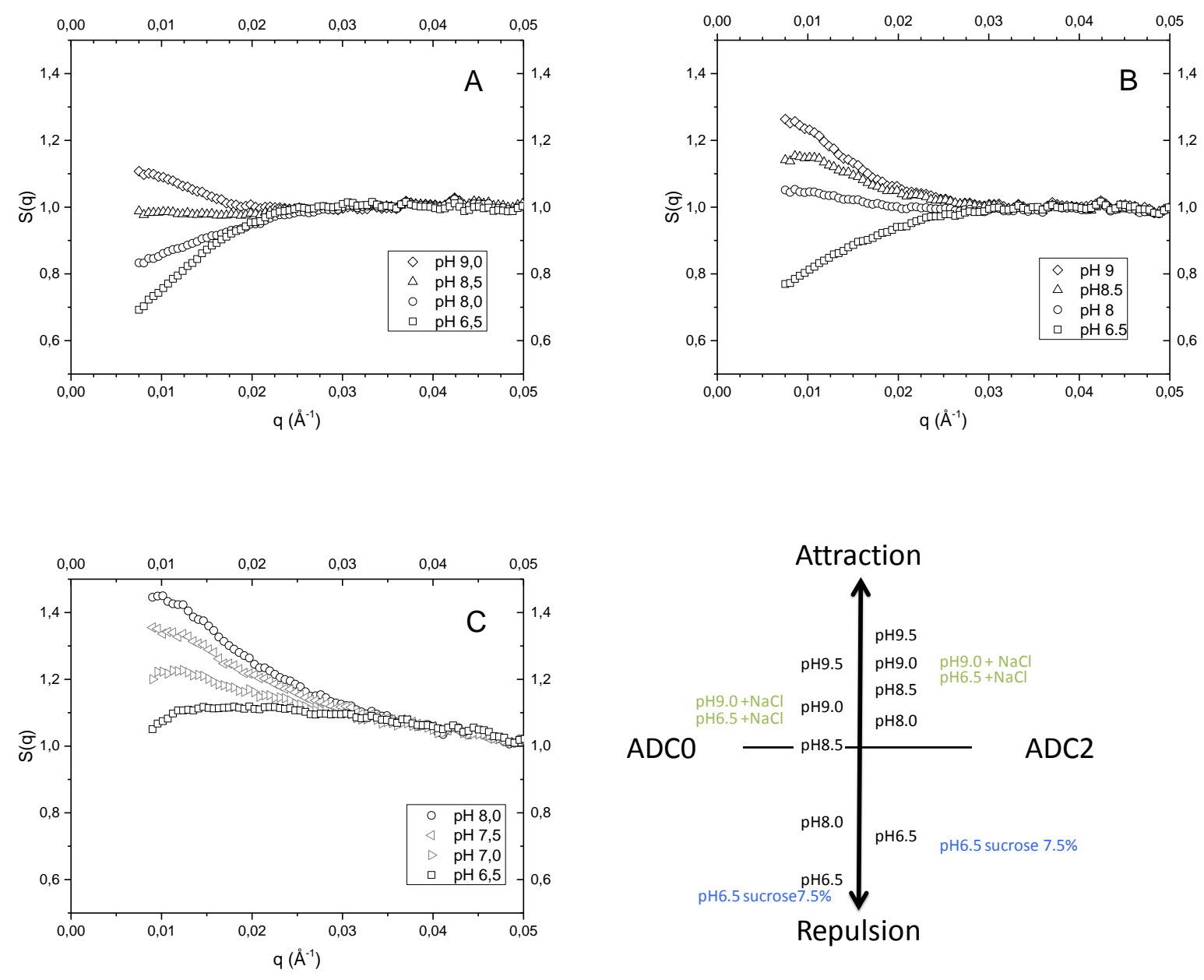

Figure 8. Variation with $\mathrm{pH}$ of inter-ADC interactions as indicated by scattering spectra at low $q$. Representation of $S_{a p p}(q)=I(q) / P(q)$ for $\mathrm{ADC}$ in $10 \mathrm{mM}$ Histidine- $\mathrm{HCl}$ solutions. (A) 4.35 g.L. $\mathrm{L}^{-1} \mathrm{ADC} 0$, (B) $4.35 \mathrm{~g} . \mathrm{L}^{-1}$ ADC2, (C) 5 g.L $\mathrm{L}^{-1}$ ADC3; (D) buffer conditions qualitatively ranked in the order of increasing mean interprotein interaction, as estimated from the rate of low$q$ variation of $S_{a p p}$ in Figures 8A-C, and Figure SI8 in ESI. The horizontal black line indicates the boundary of vanishing mean interaction. 
SAXS from ADC solutions prepared at $\mathrm{pH}>6.5$ were recorded on samples that were not filtered, and thus included contributions from aggregates. The absence of experimentally significant differences in the high- $q\left(>0.025 \AA^{-1}\right)$ scattering of ADC2 at $\mathrm{pH} 6.5$ and $\mathrm{pH} 8$ suggests that aggregation did not introduce detectable correlations between the molecules. This is a hint speaking in favor of $i$ ) preservation of ADC native-like shape (at the resolution of SAXS), and ii) absence of specific orientation between concomitant proteins in aggregates. To estimate the magnitude of deformation on IgG monomers that could be detected by SAXS, we generated model structures based on pdb crystal structures subjected to relative rotations of the Fab vs Fc domains (see experimental section). The pdb file 1IGT was somewhat arbitrarily chosen among available crystal structures as a starting scaffold, essentially because SAXS profile of unmodified 1IGT was closer to $P(q)$ of ADC0 in the high q range (Fig. SI9 in SI). SAXS scattering curves were then calculated for each distorted structure using CRYSOL routine of the ATSAS package. ${ }^{23}$ Calculated SAXS profiles revealed that the more obvious indications of relative rotations between the domains could be seen in the $q$-range between 0.1 and $0.2 \AA^{-1}$ (Figure 9A). The impact of shape distortion has been exploited in advanced structural characterization based on generation of ensembles of antibody structures to fit high-q SAXS profiles. ${ }^{22}$ Our present illustration is also consistent with the model proposed by Yearley and coll., who calculated the form factor based on a robust Y-shaped antibody-like structure (providing general trends that do not depends on a specific crystallographic structure). ${ }^{21}$ It was demonstrated in this work that variation of the angles between the two Fab domains, or between one Fab and the Fc domain, is reflected by significant shifts at $\mathrm{q}<0.08 \AA^{-1}$, and also clear variations of the slope around inflexion points present in the $0.08-0.2 \AA^{-1}$ window. By comparing the theoretical profiles in Figure $9 \mathrm{~A}$, we estimated that rotation by $20^{\circ}$ or more of one single domain is likely to be 
detected by SAXS. Figure 9B shows the comparison of experimental SAXS profiles for ADC0, $\mathrm{ADC} 2$ and $\mathrm{ADC} 3$ in this range of $q$. The similarity of all three profiles zoomed on the relevant $q$ range suggests that the relative orientation and position of $\mathrm{Fab}$ and $\mathrm{Fc}$ domains were similar in $\mathrm{ADC} 0, \mathrm{ADC} 2$ and $\mathrm{ADC} 3$.
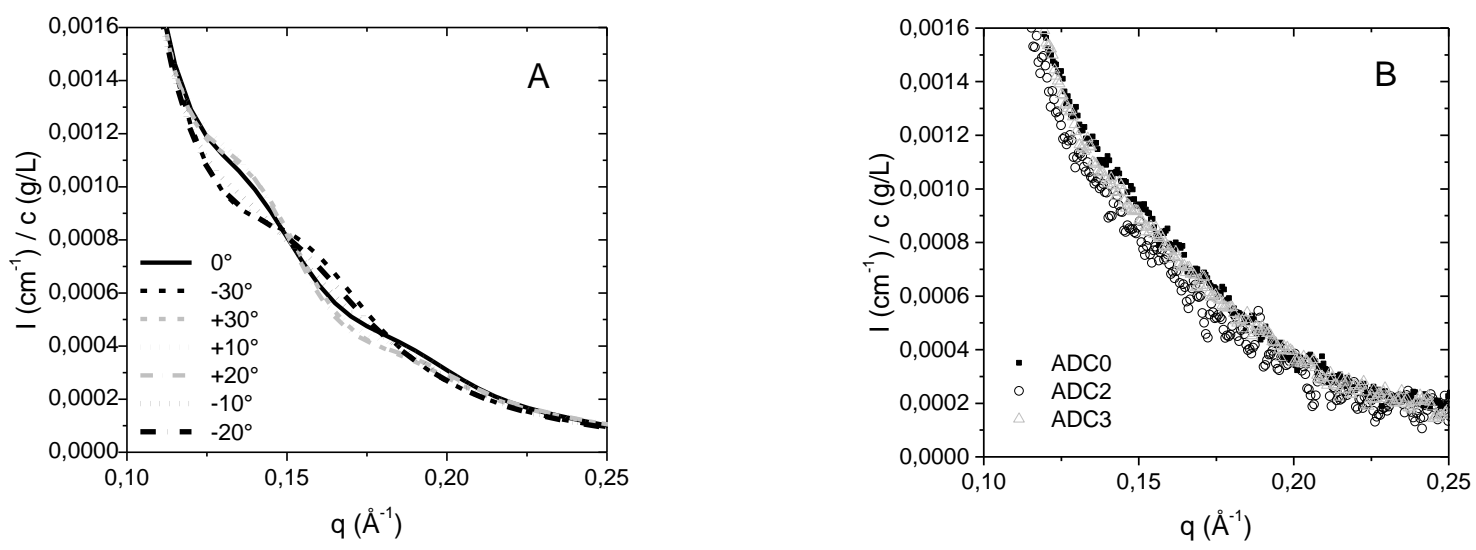

Figure 9. SAXS profiles zoomed in the $q$ range where modifications of the tertiary structure are expected to significantly change the profile. (A) Calculated SAXS profiles of model structures generated from pdb 1IGT file and distorted by rotation of one Fab region relative to the Fc domain (see experimental section and Figure SI2 in ESI), (B) experimental SAXS data recorded for ADCs.

The absence of significant change in the structural features of ADC when they were subjected to $\mathrm{pH}$ stress was assessed in addition by circular dichroism measurements. The CD spectra recorded immediately after dilution at $\mathrm{pH} 6.5,7.4$, or 7.7 did not show statistically significant differences (Figure 10). At $\mathrm{t}=1 \mathrm{~h}$, when aggregates were formed, the absence of significant change in $\mathrm{CD}$ spectra indicated that the secondary structure was not evolving in this time window. Due to the high absorbance of Histidine in UV, phosphate buffer (with $10 \%$ sucrose) was used in $\mathrm{CD}$ experiments. This modification did not qualitatively modify the propensity to 
aggregate, though we noticed that aggregation was slower in phosphate compared to Histidine buffers. abrupt, stress-induced, increase of apparent hydrodynamic radius in Fig SI3, while no change is detected at the molecular level (CD spectra). This result differs from what is generally reported in mAb solution. For instance, measurements on acidic $\mathrm{pH}$-stressed mAbs, as reported by B. Veestergaard, ${ }^{22}$ show a significant loss of monomers while denatured oligomers and HMWS become predominant. In ADC however, the predominant scattered intensity goes with a minor fraction, about 20\%, of the ADC undergoing aggregation (Table 2). Preservation of monomer in the soluble, non-aggregated, population is confirmed by stability of radii $<12 \mathrm{~nm}$ after filtration (Fig SI4 in SI). In contrast to mAbs solutions, application of a stress has presumably a variable impact on the different molecules of ADC present in the same solution, which result in " lack " of denaturation of most ADC molecules.
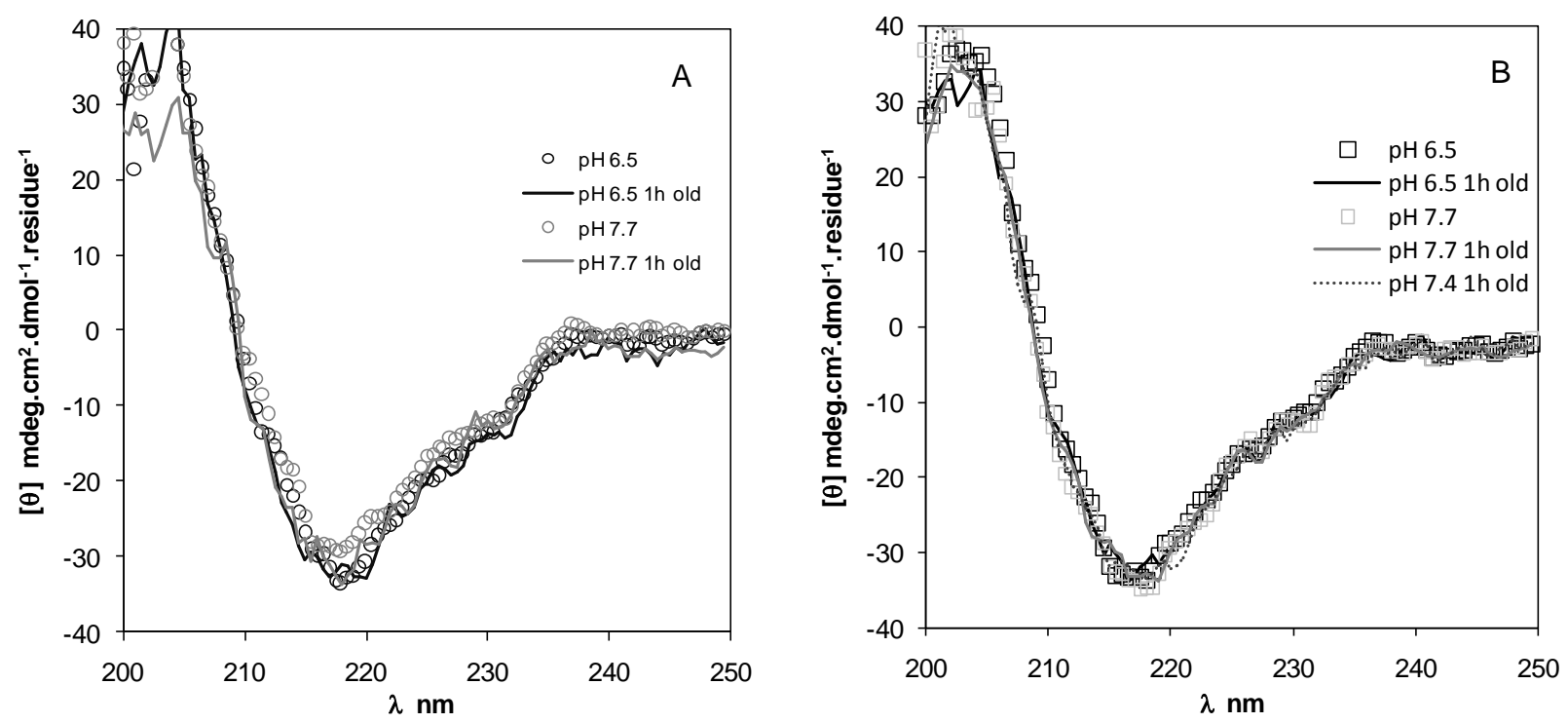

Figure 10. Circular dichroism spectra of ADC diluted at time zero in $10 \mathrm{mM} \mathrm{Na} 2 \mathrm{HPO}_{4}$ $\mathrm{NaH}_{2} \mathrm{PO}_{4}, 10 \%$ sucrose stress buffers . (A) 0.12 g.L $\mathrm{L}^{-1}$ ADC0; (B) 0.188 g.L - $^{-1}$ ADC2. "pH6.5" or "pH 7.7" stand for data recorded immediately after dilution (scans duration 3 min.), "1h old" indicates spectra recorded one hour after dilution. 
Discussion.

\section{Kinetic stability vs mean colloidal interactions}

It is generally proposed that well below temperature conditions required for unfolding, here near or below room temperature, the mean interactions between proteins in solution correlate with propensity to form aggregates. Average interaction, or virial coefficients, are used as direct criteria to predict the stability of $\mathrm{mAb}$ (see for instance relationship with phase transition studies, ${ }^{24,25}$ SAXS or SANS measurements, ${ }^{18,21,26}$ predictions based on colloidal stability ${ }^{27}$ ). General models based on Fuchs stability ratio and correlation with inter-protein attraction/repulsion have accordingly been reported to apply to mAb cold-set aggregation by Morbidelli et al. ${ }^{9,11}$ Alternative origins of instability essentially involve conformational transitions, near conditions of structural destabilization of the native fold (mechanisms based on non-native aggregation, such as in amyloid aggregation ${ }^{28}$ ). Conformational instability is typically expected to be significant in $\mathrm{mAb}$ aggregation above $40^{\circ}-50^{\circ} \mathrm{C} \cdot{ }^{29,30,31,32,33}$ (for a recent review on aggregation mechanisms see ${ }^{34}$ ) In the present study, kinetics data lacked the signature of conformational transition (no lag time nor nucleation regime, no evidence for autocatalysis), which justified the use of the colloidal stability model.

Maximum aggregation propensity was reached in the $\mathrm{pH}$ range 7 to 8 for $\mathrm{ADC} 2$ and $\mathrm{ADC} 3$, as reflected by the smooth minimum in $W_{\text {eff }}$ near $\mathrm{pH}$ 7.8. In contrast, mean inter-protein interactions reflected by SAXS spectra appeared to vary monotonously from weakly repulsive to attractive with increasing $\mathrm{pH}$ from 6.5 to 8.5, suggesting that maximum attraction between ADCs was reached near or above $\mathrm{pH}$ 8.5-9.0. Somewhat surprisingly, the evolution toward attractive 
interactions by increasing the $\mathrm{pH}$ above 8 was accompanied by an increase of $W_{\text {eff }}$, i.e. stronger average attractions above $\mathrm{pH} 8$ corresponded to slower aggregation kinetics. Other apparent inconsistency between interaction pattern and kinetic stability include the stabilizing effect of added $\mathrm{NaCl}$ at $\mathrm{pHs}$ far from the $\mathrm{pH}$ of maximum attractive interaction (i.e. far from the isoelectric point), where Coulomb repulsions should limit interprotein collisions. Mean attraction between ADC is accordingly NOT predictive of the kinetics of aggregation. Polydispersity is the major difference between $\mathrm{ADC}$ and model solutions of mAb. Covalent coupling of mAb with the drug molecules yielded here a mixture of proteins having diverse drug:mAb ratios, and a variety of possible surface distribution of the conjugation points, including uneven ones that may locally form densely modified patterns. As opposed to SAXS (that give access to average interactions over the whole population of ADC), kinetics of aggregation is primarily sensitive to the unstable (sub)fraction of ADCs. Apparent inconsistencies between variations of the average interaction parameters and kinetics of aggregation, together with our measurements of the amount of aggregates, usually $<30 \%$ of the total protein, reveals the specific contribution to instability of a subpopulation of ADCs. The predominant contribution of an unstable fraction is also consistent with the high stability reached when samples were filtered a few second after application of the $\mathrm{pH}$ stresses (removal of the unstable fraction).

Influence of Lysine modification onto surface charge distribution on ADC

We illustrate in this section the presence of patches of opposite charges on ADC, that was inferred from the increase of $W^{*}$ with increasing $\mathrm{NaCl}$ at $\mathrm{pH} \sim 7-8$. Figure 11 shows various $\mathrm{mAb}$ configurations that favor or not the protrusion of local negatively-charged patches upon 
heterologous substitution in ADC0 of two positively-charged Lysine residues by a neutral Leucine ones (model calculated at $\mathrm{pH} 7.8$, see experimental section). The impact of the substitution of two Lysine residues on Coulomb potential was generally weak if the residues were embedded in a positively charged region (Figure 11B). In contrast, modification of two Lysines could turn a weakly charged region into an obvious anionic, protruding pole (Figure 11C) with a range of negative potential similar to the highest positive protrusions seen in Fab domains. The estimated isolectric point did not shift by more than $0.1 \mathrm{pH}$ units, from 8.89 down to 8.79 in all the configurations tested (Figure 11D). These purely qualitative and arbitrary considerations confirm that a small number of modifications on Lysine are sufficient to create charge configurations on $\mathrm{ADC}$ that display both positive and negative regions at $\mathrm{pH} 7.8$, while being well below the global isoelectric point. Therefore, we propose that stabilization of ADC in the presence of $\mathrm{NaCl}$, a behavior that was observed experimentally at $\mathrm{pH} \sim 7-8$, presumably involves the decrease of Coulomb attractions between positively and negatively charged patches, i.e. an electrostatic surface potential markedly differing from the charge pattern of ADC0 (N.B.: this does not preclude that the charged patches may also be hydrophobic, due to attachment of the drug). The hypothesis of the involvement of ionic patches in inter-protein bridging is not new. It has been reported in reversible phase transition (coacervation) of native globular proteins. ${ }^{35}$ Recent studies suggest that coulomb attraction can also favor irreversible aggregation of monoclonal and polyclonal antibodies. ${ }^{36,37,38}$ The main difference with previous studies relies here on the fact that in the case of ADCs, patches are due to the chemical modification and their number and composition are accordingly governed by statistic distribution of the modified residues. 


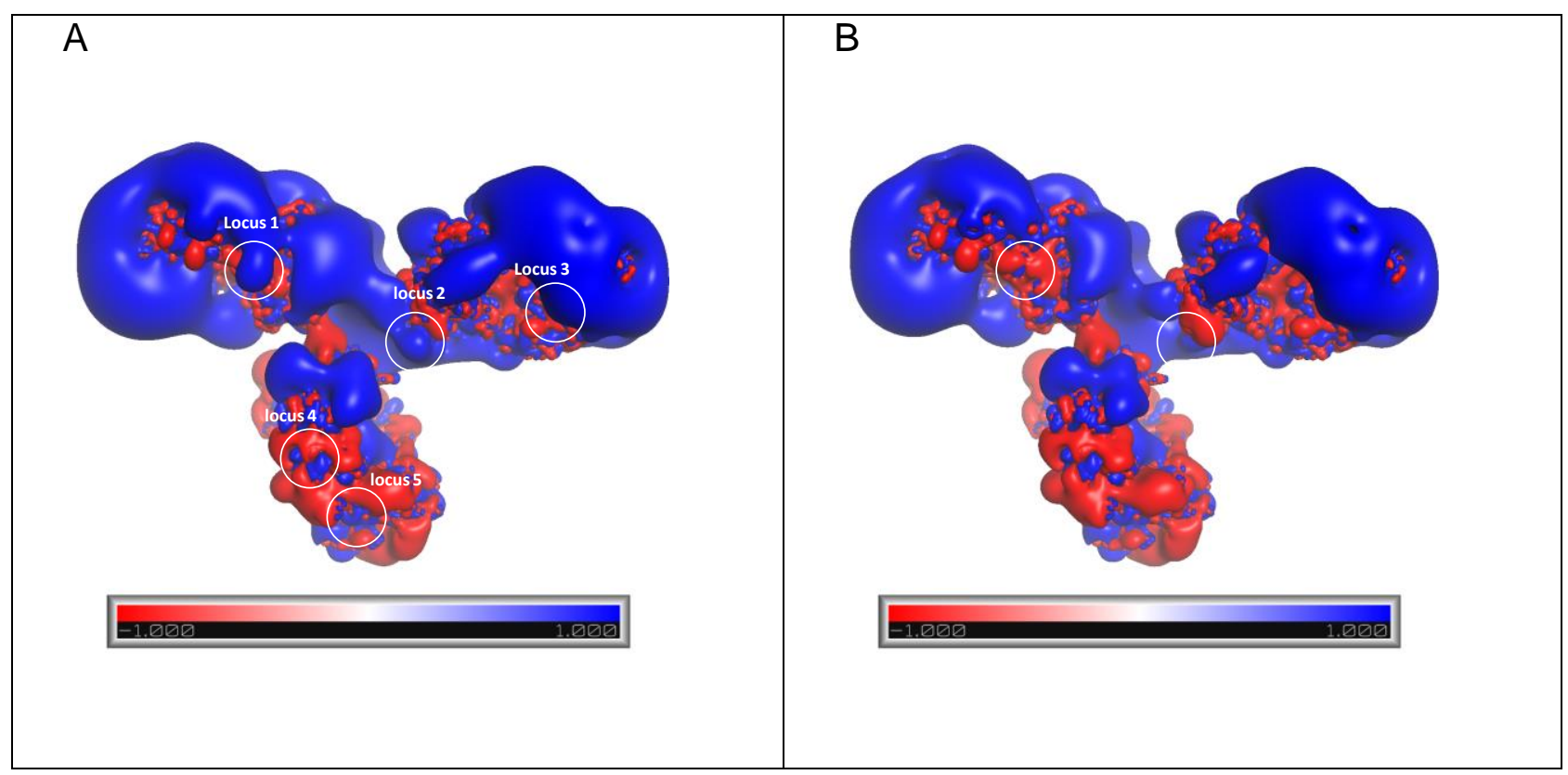




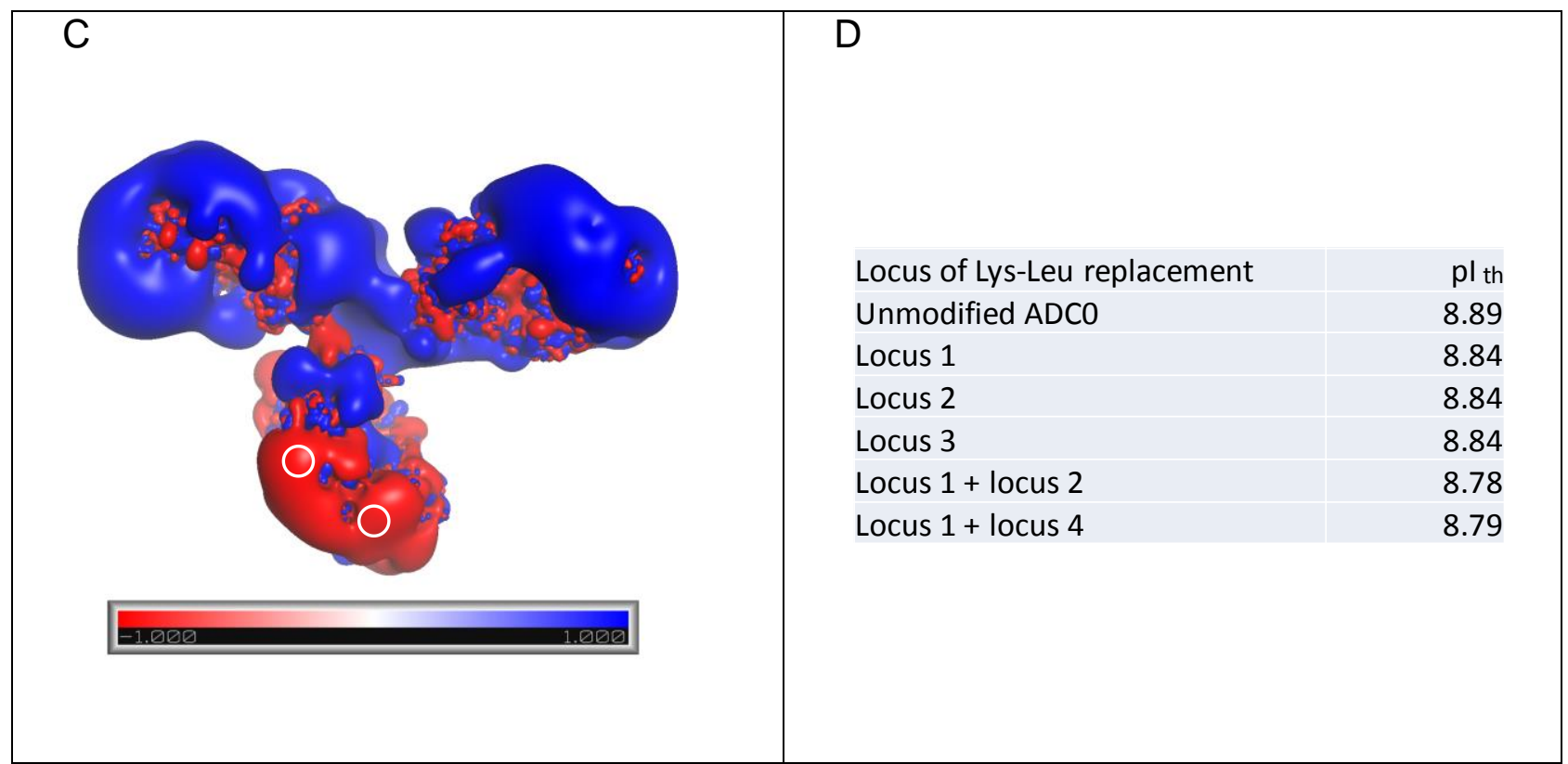

Figure 11. Electrostatic surface isocontour $\left(+/-1 \mathrm{k}_{\mathrm{B}} \mathrm{T} / \mathrm{e}\right)$ of $\mathrm{mAb}(\mathrm{ADC} 0)$ calculated using Adaptative Poisson-Boltzman Solver software with $10 \mathrm{mM}$ ionic strength and $\mathrm{pH} 7.8$ as parameters. (A) Unmodified ADC0 and identification by circles of Lysine residues located in regions of near-neutral or weakly negative mean charge. (B) Illustration of an isopotential of a doubly-modified ADC (Lysine replaced by Leucine in loci 1 and 2), i.e. near positive "blue" protrusions in Fab domains (similar results were obtained with pairs of modifications at the loci 1, 2 or 3 shown in Figure 11A); (C) Illustration of an isopotential of a doubly-modified ADC where replacement of Lysine by Leucine were made at loci 4 and 5. (D) pI calculated with the "propKa software" package. ${ }^{16,15}$

Conclusions.

Results from light scattering and SAXS on antibody-drug conjugates established the distinct influence of composition parameters of the solution ( $\mathrm{pH}$, ionic strength, sucrose) on roomtemperature aggregation rate and interprotein interactions. In particular, determination of ADC kinetic stability and of average interADC interactions as a function of $\mathrm{pH}$, and with or without $\mathrm{NaCl}$ in buffers, suggest that the rate of formation of ADC clusters can be maximal under 
conditions corresponding to weak average repulsions between the proteins. The conjugation of monodisperse monoclonal antibody introduces a degree of polydispersity in ADC that is proposed to be at the origin of the counterintuitive observation of a minimal stability at $\mathrm{pH}$ conditions corresponding experimentally to average repulsions, or weak interactions between ADC. Involvement of a pH-dependent subset of the whole population of ADC is validated by measurement of the fraction of aggregated proteins that typically reached plateaus below 20$35 \mathrm{~mol} \%$, and by the high, days-long stability of the filtrates obtained just after a $\mathrm{pH}$-triggered destabilization (containing the majority of the initial ADCs). Overall, this study demonstrates that ADC polydispersity (here due to the number and distribution of chemically modified Lysines) should not be overlooked, particularly when developing formulation of therapeutic antibody-drug conjugates. On more fundamental grounds, protein conjugates having a structural diversity due to the variability of discrete, residue-specific, surface modifications provide a new experimental model that opens interesting questions in the field of colloid aggregation.

Supporting Information. SEC analysis of ADC, images of distorted IgG, UV-vis. absorption spectra before and after filtrations, light scattering data, SAXS spectra are available free of charge via the Internet at http://pubs.acs.org.

\section{Corresponding Author}

* christophe.tribet@ens.fr

\section{Present Addresses}

†University of Cambridge (UK), Dept of Chemistry ; ₹ University of Bristol, Dept of Chemistry. 


\section{Author Contributions}

The manuscript was written through contributions of all authors. All authors have given approval to the final version of the manuscript.

\section{ACKNOWLEDGMENT}

This work was supported by the French National Research Agency (program Blanc International, grant ANR 2010-INT 1501, and program Investissement d'Avenir ANR-11LABX-0011-01, and by SANOFI research grant to BFP. Authors are grateful to Javier Perez and Aurélien Thureau for their help and advice in SAXS measurements at SOLEIL. We thank Sophie Norvez from MMC laboratory in ESPCI for her help with circular dichroism.

\section{REFERENCES}

1. Leader, B.; Baca, Q.J.; Golan, D.E. Protein therapeutics: a summary and pharmacological classification. . Nat. rev., Drug Discov. 2008, 7, 21-39. .

2. Biologic drugs set to top 2012 sales. Nat. Med. 2012, 18 (5), 636-636.

3. Ma, L.L.; Tam, J.O.; Willsey, B.W.; Rigdon, D.; Ramesh, R.; Sokolov, K.; Johnston, K.P. Selective Targeting of Antibody Conjugated Multifunctional Nanoclusters (Nanoroses) to Epidermal Growth Factor Receptors in Cancer Cells. Langmuir 2011, 27 (12), 7681-7690.

4. Chari, R.V.; Miller, M.L.; Widdison, W.C. Antibody-drug conjugates: An emerging concept in cancer therapy. Angew. Chem. Int. Ed. 2014, 53, 3796-3827.

5. Roberts, C.J.; Das, T.K.; Sahin, E. Predicting solution aggregation rates for therapeutic proteins: Approaches and challenges. Int. J. Pharm. 2011, 418 (2), 318-333.

6. $\quad$ Brummitt, R.K.; Nesta, D.P.; Roberts, C.J. Predicting Accelerated Aggregation Rates for Monoclonal Antibody Formulations, and Challenges for Low-Temperature Predictions. $J$. Pharm. Sci. 2011, 100 (10), 4234-4243.

7. Castellanos, M.M.; Pathak, J.A.; Colby, R.H. Both protein adsorption and aggregation contribute to shear yielding and viscosity increase in protein solutions. Soft Matter 2014, 10 (1), 122-131. 
8. Rudiuk, S.; Cohen-Tannoudji, L.; Huille, S.; Tribet, C. Importance of the dynamics of adsorption and of a transient interfacial stress on the formation of aggregates of IgG antibodies. Soft Matter 2012, 8 (9), 2651-2661.

9. Owczarz, M.; Motta, A.C.; Morbidelli, M.; Arosio, P. A Colloidal Description of Intermolecular Interactions Driving Fibril-Fibril Aggregation of a Model Amphiphilic Peptide. Langmuir 2015, 31 (27), 7590-600.

10. Wu, H.; Xie, J.J.; Morbidelli, M. Kinetics of cold-set diffusion-limited aggregations of denatured whey protein isolate colloids. Biomacromolecules 2005, 6 (6), 3189-3197.

11. Arosio, P.; Rima, S.; Lattuada, M.; Morbidelli, M. Population Balance Modeling of Antibodies Aggregation Kinetics. J. Phys. Chem. B 2012, 116 (24), 7066-7075.

12. Bouchard, H.; Commercon, A.; Fromond, C.; Mikol, V.; Parker, F.; Sassoon, I.; Tavares, D. Antibodies that specifically bind to EPHA2 receptor 2014, US patent 2014, US 8,668,910 B2.

13. Dolinsky, T.J.; Nielsen, J.E.; McCammon, J.A.; Baker, N.A. PDB2PQR: an automated pipeline for the setup of Poisson-Boltzmann electrostatics calculations. Nucleic Acids Res. 2004, 32, W665-W667.

14. Baker, N.A.; Sept, D.; Joseph, S.; Holst, M.J.; McCammon, J.A. Electrostatics of nanosystems: Application to microtubules and the ribosome. Proc. Natl. Acad. Sci. USA 2001, 98 (18), 10037-10041.

15. Sondergaard, C.R.; Olsson, M.H.M.; Rostkowski, M.; Jensen, J.H. Improved Treatment of Ligands and Coupling Effects in Empirical Calculation and Rationalization of pK(a) Values. J. Chem. Theory Comput. 2011, 7 (7), 2284-2295.

16. Olsson, M.H.M.; Sondergaard, C.R.; Rostkowski, M.; Jensen, J.H. PROPKA3: Consistent Treatment of Internal and Surface Residues in Empirical pK(a) Predictions. J. Chem. Theory Comput. 2011, 7 (2), 525-537.

17. Timasheff, S.N. Protein hydration, thermodynamic binding, and preferential hydration. Biochemistry 2002, 41 (46), 13473-13482.

18. Castellanos, M.M.; Pathak, J.A.; Leach, W.; Bishop, S.M.; Colby, R.H. Explaining the Non-Newtonian Character of Aggregating Monoclonal Antibody Solutions Using Small-Angle Neutron Scattering. Biophys. J. 2014, 107 (2), 469-476.

19. Lilyestrom, W.G.; Shire, S.J.; Scherer, T.M. Influence of the Cosolute Environment on IgG Solution Structure Analyzed by Small-Angle X-ray Scattering. J. Phys. Chem. B 2012, 116 (32), 9611-9618.

20. Lilyestrom, W.G.; Yadav, S.; Shire, S.J.; Scherer, T.M. Monoclonal Antibody SelfAssociation, Cluster Formation, and Rheology at High Concentrations. J. Phys. Chem. B 2013, 117 (21), 6373-6384.

21. Yearley, E.J.; Zarraga, I.E.; Shire, S.J.; Scherer, T.M.; Gokarn, Y.; Wagner, N.J.; Liu, Y. Small-Angle Neutron Scattering Characterization of Monoclonal Antibody Conformations and Interactions at High Concentrations. Biophys. J. 2013, 105 (3), 720-731.

22. Skamris, Th.; Tian, X.; Thorolfsson, M.;Karkov H. S.; Rasmussen H.B.; Langkilde A. E.; Vestergaard B. Monoclonal Antibodies Follow Distinct Aggregation Pathways During Production-Relevant Acidic Incubation and Neutralization. Pharm Res 2016, 33:716-728.

23. Petoukhov, M.V.; Franke, D.; Shkumatov, A.V.; Tria, G.; Kikhney, A.G.; Gajda, M.; Gorba, C.; Mertens, H.D.T.; Konarev, P.V.; Svergun, D.I. New developments in the ATSAS program package for small-angle scattering data analysis. J. Appl. Cryst. 2012, 45, 342-350. 
24. Wang, Y.; Latypov, R.F.; Lomakin, A.; Meyer, J.A.; Kerwin, B.A.; Vunnum, S.; Benedek, G.B. Quantitative Evaluation of Colloidal Stability of Antibody Solutions using PEGInduced Liquid-Liquid Phase Separation. Molecular Pharm. 2014, 11 (5), 1391-1402.

25. Wang, Y.; Lomakin, A.; Latypov, R.F.; Laubach, J.P.; Hideshima, T.; Richardson, P.G.; Munshi, N.C.; Anderson, K.C.; Benedek, G.B. Phase transitions in human IgG solutions. $J$. Chem. Phys. 2013, 139 (12).

26. Tessier, P.M.; Wu, J.; Dickinson, C.D. Emerging methods for identifying monoclonal antibodies with low propensity to self-associate during the early discovery process. Expert Opin. Drug Deliv. 2014, 11 (4), 461-465.

27. Arzensek, D.; Kuzman, D.; Podgornik, R. Colloidal interactions between monoclonal antibodies in aqueous solutions. J. Colloids Interf. Sci. 2012, 384, 207-216.

28. Murray, B.; Rosenthal, J.; Zheng, Z.; Isaacson, D.; Zhu, Y.; Belfort, G. Cosolute Effects on Amyloid Aggregation in a Nondiffusion Limited Regime: Intrinsic Osmolyte Properties and the Volume Exclusion Principle. Langmuir 2015, 31 (14), 4246-4254.

29. Arosio, P.; Jaquet, B.; Wu, H.; Morbidelli, M. On the role of salt type and concentration on the stability behavior of a monoclonal antibody solution. Biophys. Chem. 2012, 168, 19-27.

30. Sahin, E.; Grillo, A.O.; Perkins, M.D.; Roberts, C.J. Comparative Effects of pH and Ionic Strength on Protein-Protein Interactions, Unfolding, and Aggregation for IgG1 Antibodies. $J$. Pharm. Sci. 2010, 99 (12), 4830-4848.

31. Thakkar, S.V.; Sahni, N.; Joshi, S.B.; Kerwin, B.A.; He, F.; Volkin, D.B.; Middaugh, C.R. Understanding the relevance of local conformational stability and dynamics to the aggregation propensity of an IgG1 and IgG2 monoclonal antibodies. Protein Sci. 2013, 22 (10), 1295-1305.

32. Brummitt, R.K.; Nesta, D.P.; Chang, L.Q.; Chase, S.F.; Laue, T.M.; Roberts, C.J. Nonnative Aggregation of an IgG1 Antibody in Acidic Conditions: Part 1. Unfolding, Colloidal Interactions, and Formation of High-Molecular-Weight Aggregates. J. Pharm. Sci. 2011, 100 (6), 2087-2103.

33. Broersen, K.; Weijers, M.; de Groot, J.; Hamer, R.J.; de Jongh, H.H.J. Effect of protein charge on the generation of aggregation-prone conformers. Biomacromolecules 2007, 8 (5), 1648-1656.

34. Amin, S.; Barnett, G.V.; Pathak, J.A.; Roberts, C.J.; Sarangapani, P.S. Protein aggregation, particle formation, characterization \& rheology. Curr. Opin. Colloid Interf. Sci. 2014, $19(5), 438-449$.

35. Yan, Y.; Seeman, D.; Zheng, B.; Kizilay, E.; Xu, Y.; Dubin, P.L. pH-Dependent Aggregation and Disaggregation of Native beta-Lactoglobulin in Low Salt. Langmuir 2013, 29 (14), 4584-4593.

36. Brunsteiner, M.; Flock, M.; Nidetzky, B. Structure Based Descriptors for the Estimation of Colloidal Interactions and Protein Aggregation Propensities. Plos One 2013, 8 (4).

37. Martin, N.; Ma, D.; Herbet, A.; Boquet, D.; Winnik, F.M.; Tribet, C. Prevention of Thermally Induced Aggregation of IgG Antibodies by Noncovalent Interaction with Poly(acrylate) Derivatives. Biomacromolecules 2014, 15 (8), 2952-62.

38. Yadav, S.; Shire, S.J.; Kalonia, D.S. Viscosity behavior of high-concentration monoclonal antibody solutions: Correlation with interaction parameter and electroviscous effects. J. Pharm. Sci. 2012, 101 (3), 998-1011. 
TOC graphic:

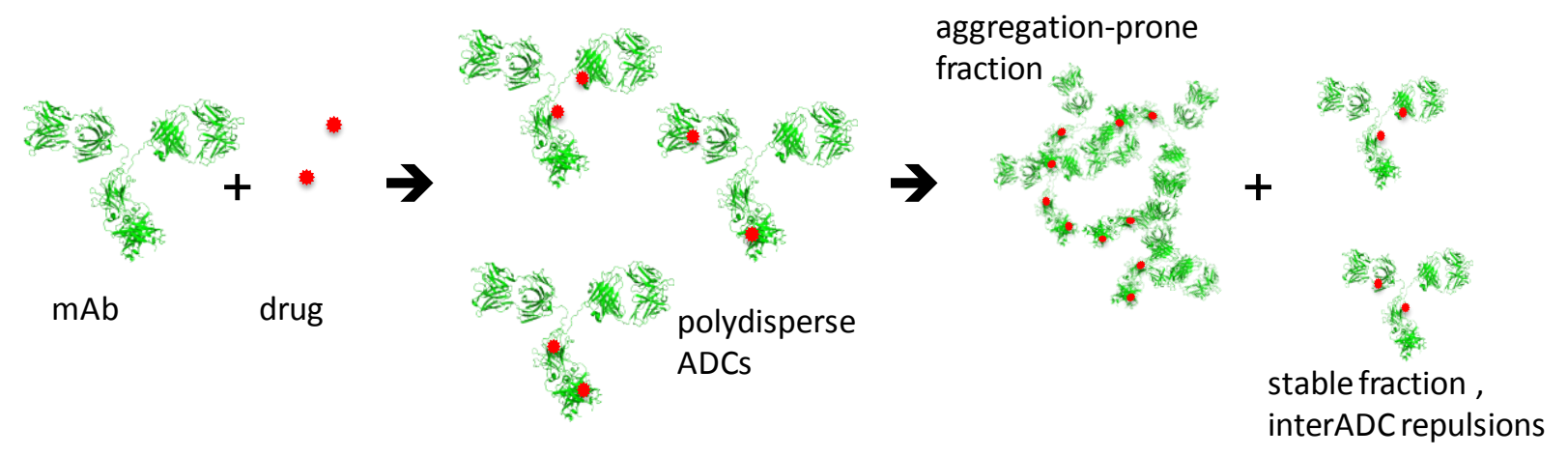

